\title{
An Intelligent Feature Selection using Archimedes Optimization algorithm for Facial Analysis
}

Imène NEGGAZ ( $\nabla$ imene.neggaz@univ-usto.dz )

Universite des Sciences et de la Technologie d'Oran Mohamed Boudiaf https://orcid.org/0000-00028673-7710

Hadria FIZAZI

Universite des Sciences et de la Technologie d'Oran Mohamed Boudiaf

\section{Research Article}

Keywords: Archimedes optimization algorithm (AOA), Human facial analysis (HFA), Wrapper Feature Selection (FS), Handcrafted methods, Automatic selection

Posted Date: July 19th, 2021

DOI: https://doi.org/10.21203/rs.3.rs-636151/v1

License: (c) (i) This work is licensed under a Creative Commons Attribution 4.0 International License. Read Full License 


\title{
An Intelligent Feature Selection using Archimedes Optimization algorithm for Facial Analysis
}

\author{
Imène Neggaz ${ }^{\mathrm{a}}$, Hadria Fizazi ${ }^{\mathrm{a}}$ \\ ${ }^{a}$ Université des Sciences et de la Technologie d'Oran Mohamed Boudiaf, USTO-MB, BP 1505, EL M'naouer, \\ 31000 Oran-Algérie \\ - Laboratoire Signal Image PArole (SIMPA)-Département d'informatique \\ Faculté des Mathématiques et Informatique \\ Email: imene.neggaz@univ-usto.dz,hadriafizazi@yahoo.fr
}

Email address: imene.neggaz@univ-usto.dz (corresponding author) (Imène Neggaz) 
Abstract. Human facial analysis (HFA) has recently become an attractive topic for computer vision research due to the technological progress and the increase of mobile applications. HFA explores several issues as gender recognition, facial expression, age, and race recognition for automatically understanding social life. In addition, the development of several algorithms inspired by swarm intelligence, biological inspiration, and physical/mathematical rules allow giving another dimension of feature selection in the field of machine learning and computer vision.

This paper develops a novel wrapper feature selection method for gender recognition using the Archimedes optimization algorithm (AOA). The paper's primary purpose is to automatically determine the optimal face area using AOA to recognize the gender of a human person categorized by two classes (Men and women). In this paper, the facial image is divided into several sub-regions (blocks), where each area provides a vector of characteristics using one method from handcrafted techniques as the local binary pattern (LBP), histogram oriented gradient (HOG), or Grey level co-occurrence matrix (GLCM). The proposed method (AOA) is assessed on two publicly datasets: Georgia Tech Face dataset (GT) and the Brazilian FEI dataset. The experimental results show a good performance of AOA compared to other recent and competitive optimizers as Sine cosine algorithm (SCA), Henry Gas Solubility Optimization (HGSO), Equilibrium Optimizer (EO), Emperor Penguin Optimizer (EPO), Harris Hawks Optimize (HHO), Multi-verse Optimizer (MVO) and Manta-ray Foraging Optimizer (MRFO) in terms of accuracy and the number of the selected area.

Keywords: Archimedes optimization algorithm (AOA); Human facial analysis (HFA); Wrapper Feature Selection (FS); Handcrafted methods; Automatic selection.

\section{Introduction}

Human vision allows performing several tasks in parallel and a rapid time, particularly facial detection, gender recognition, and recognizing the state of mind, which differentiates the human being from others.

The automation of gender recognition represents a real challenge for scientific researchers, and it has a significant impact on the commercial field and video surveillance. For example, shopping centers are interested in knowing the sales rate and the category of people who buy their products, particularly the gender, age, and origins, to increase the sales rate. Also, another area requires the application of gender recognition to detect suspected people, captured by surveillance cameras in large spaces such as airports, shopping malls, and gas stations. In order to reduce the time of searching for the target suspected person, the gender recognition application can contribute profoundly to solving this issue, especially for critical situations as suicide bombing or airport attack. Also, the current situation of the Covid-19 pandemic obliged people to wear the mask that the automation of gender recognition plays a vital role in our life.

According to the literature, the human detection rate is lower than $95 \%$, which increases the difficulties of automatic gender recognition. In this field, several challenges are considered as rotated, occluded faces, and the human who is similar to women with long hair, which strongly affects the performance of gender recognition.

In general, the task of gender recognition required two crucial steps. The first step aims to extract the features from faces, while the second step is reserved for realizing the task of classification into binary classes using machine learning approaches as Support Vector Machines (SVM), Extreme Learning Machine (ELM), and Multi-layer Perceptron (MLP). As a case study, we find the work of [1] which applied Kernel PCA for reducing the size of intensity vectors and 
providing 64 principal components for gender classification. This vector is considered as aninput of SVM that used 2-folds cross-validation on three datasets.

The quantitative study has shown a performance of accuracy $97.375 \%, 99.7 \%$ and $96.67 \%$ for the three datasets: GT, AT@T and Faces94 datasets. Several descriptors based on handcrafted are introduced for face recognition, gender, and age identification during the last decades. This type includes mainly four categories: texture features, facial shape features, intensity pixels and geometric features. The first type includes predominately local binary pattern (LBP) [2], Local phase quantization (LPQ) [3 and Local Ternary Patterns (LTP) [4 while the second type employed basically histogram oriented gradient (HOG) [5, Pyramid HOG (PHOG) and Multi-level HOG (ML-HOG) [6]. Additionally, the third type used mostly grey level co-occurrence matrix (GLCM) and Rotated GLCM. Finally, the features are extracted by scale-invariant features transform (SIFT). It is important to highlight that a great number of algorithms are designed in order to enhance the basic handcrafted methods by fusing textural information with facial shape features for gender recognition [7]. This work consists of extracting two textural descriptors ( Dominated RLBP and Rotation Invariant LPQ), combined with Pyramid HOG, to determine the gender of persons automatically. In addition, the authors employed an SVM classifier based on three kernel functions (Linear, Polynomial and RBF). The experimental study has been validated by three datasets: FEI, LFW and Adience. The obtained results proved that the SVM based RBF kernel achieved higher performance in terms of accuracy for FEI, LFW and Adience with 95.3\%, 98.7\% and $96 \%$, respectively. In the same context, 8 designed a new gender descriptor which is based on edge feature, texture feature and intensity characteristics. The first part is encoded by 8-Local directional pattern (8-LDP), whereas LBP implements the second part and the final part represents the pixels values of the image and the final part represents the pixels values of the image. The authors used two datasets: FEI and a self-designed dataset for treating gender classification tasks. The obtained results attained $99 \%$ as accuracy for FEI and $94 \%$ for self-designed dataset using an SVM classifier based on multi-block combined descriptor .

Nowadays, deep learned features are used exponentially in machine learning, especially in computer vision [9], biomedical application [10, 11] and remote sensing [12, 13]. Recently, several architecture are created by pretrained CNN such as VGG16, ResNet, GoogleNet and CaffeNet for gender recognition [14, 15, 16, 17, 18], AlexNet[19], Inception [20]. Furthermore, a comparative study between several pretrained CNN asuch s MobileNet, DensNet, Xception and SqueezeNet is realized by [21] for gender recognition. So, a great competition between handcrafted features and deep learned features has been highlighted. For instance, we find the work of [16], which used three pretrained CNN called CaffeNet, VGG16 and GoogleNet for estimating age and gender information.

The great number of attributes generated by both methods (Handcrafted and deep features) prompts researchers to develop new selection methods called wrapper feature selection based on meta-heuristics (MHs). MHs are derived from different subjects, allowing the development of a large number of optimization algorithms that can be merged with machine learning techniques.

In the early 1990s, researchers drew inspiration from genetic operators by creating Genetic algorithms(GA) [22], Differential evolution (DE) [23], Evolutionary strategies (ES) [24, 25] and Genetic programming (GP) [26]. Then, another axis attracts scientists, which is based on imitating the behavior of swarms by introducing the theory of swarm intelligence (SI). This category has grown so far by producing the Practical Swarm Optimzer (PSO) [27], the Artificial Bee Colony (ABC) [28], the Gray Wolf Optimizer (GWO) [29], the Harris hawks optimizer (HHO) [30], the Whale Optimization Algorithm (WOA) [31], salp swarm algorithm (SSA) [32, Grasshopper Otimization Algorithm (GOA) [33], Ant Lion Optimizer (ALO) [34, Emperor penguin optimizer 
(EPO) [35], Manta-ray Foraging Optimizer (MRFO) [36]. Also, we notice that physics and mathematical inspiration have made it possible to create new algorithms like AOA [37, Equilibrium optimizer [38, Multi-verse optimizer (MVO) [39], Henry Gas Solubility Optimization (HGSO) [40], Electromagnetism optimizer (EMO) [41, Thermal exchange optimizer (TEO) [42], Arithmetic optimization algorithm, Runge kutta optimizer (RUN) [43] and Sine cosine algorithm (SCA) [4]. More recently, game theory attracts the curiosity of scientific to develop new algorithms as Hunger games serach [45], Volleyball premier league (VPL) [46].

Despite the significant development of metaheuristics generated by the imitation of animal behavior, mathematical and physical laws in the field of feature selection as MRFO [47], EPO [48], HHO [49], GWO [50], WOA [51], HGSO [52], MVO, EO [53], and SCA [54], the integration of these methods in complex problems such as gender recognition remains limited. only a few works have been published in this area. As example, [55] used genetic algorithm (GA) to classify automatically gender based faces. Their idea consists to apply GA in order to determine the optimal set of eigen-features extracted from faces by PCA and classified by neural network.

The experimental study is validated using two datasets including FEI and FERET. The obtained results achieved $96 \%, 94 \%$ as accuracy rates, respectively.

In gender recognition from face images, a big challenge that remains to this day is how to determine the most significant areas from face images characterized by local binary pattern (LBP), histogram of oriented gradient (HOG) or Grey level co-occurrence matrix (GLCM) descriptors intelligentlly and automatically?

This paper automatically determines the significant areas based on handcrafted features (LBP, HOG, or GLCM) from the face using the Archimedes optimization algorithm (AOA) to solve gender recognition problems using an optimal number of faces extracted areas.

The major contributions of this paper are as follows:

- Designing a novel wrapper physical algorithm AOA for predicting gender identification using an automatic selection of the optimal and significant areas of face images.

- Comparing the performance of AOA with several recent and robust optimizers as for facial analysis based on FS.

- Evaluating the impact of three handcrafted features based on LBP, HOG, and GLCM.

- Testing the efficiency of AOA for gender recognition over two datasets: FEI and GT.

The following structure of our paper contains six sections. Section. 2 explains some works which treat gender recognition based on handcrafted features, deep features and hybrid descriptors. In section. 3, three handcrafted descriptors are detailled including local binary pattern (LBP), histogram oriented gradient (HOG), and Grey level co-occurrence matrix (GLCM). Section 4 gives the concept of the Archimedes optimizer algorithm in detail. After, we propose our architecture of AOA wrapper feature selection for gender recognition by defining the structure of the encoding solution of an immersed object, the score function and, the designed framework. The section represents the kernel of our paper which includes datasets description, parameters of algorithms, quantitative and graphical results proved by statistical analysis using Ranksum Wilcoxon's. Finally, section. 7. shows our conclusion with some future horizons.

\section{Related work}

This part summarizes the potential work of literature related to facial analysis. Firstly, we give a recap of handcrafted features for gender recognition. Secondly, a rapid description of deep- 
learned features is shown for human facial analysis. Thirdly, hybrid features are described. Finally, an overview of wrapper feature selection-based gender recognition is presented.

\subsection{Handcrafted features}

Recently, several methods are developed in the literature called handcrafted techniques. The extracted features are determined from the whole face or some regions by computing the local gradient parameters as a histogram of oriented gradients (HOGs) and scale-invariant feature transform (SIFT) [56]. The authors used a support vector machine as a classifier for identifying the two classes: female and male from Color FERET datasets. The experimental study has shown that HOG outperformed the SIFT descriptor-based SVM, when the size of training data is reduced.

[57 developed a novel fusion of facial features for gender recognition. The vector of characteristics is obtained by combining local binary pattern (LBP), local phase quantization (LPQ) and a multiblock. The task of classification is realized using a support vector machine and tested on Image of Group datasets (IoG). The experimental results shown that the proposed method outperformed another basic versions (LBP and LPQ).

[58] designed four frameworks for gender identification using facial images. The first framework consists of extracting features using the texture method based on LBP and reduced the dimension of vector features using PCA, which will be served as input for multi-layer perceptron (MLP).The second framework used Gabor filters to provide the vector of features reduced by PCA and served as input for the kernel SVM classifier. The third framework extracts the lower part of face with the size of sub-image $(30 \times 30)$, which will be reshaped to column vector with the size of $(900 \times 1)$ and served as input for kernel SVM classifier. The last framework consists of extracting 34 landmarks from the face, classified by linear discriminant algorithm (LDA). All proposed frameworks are assessed on FEI datasets, and the experimental results shown that the third framework outperforms others by $90 \%$ in terms of accuracy. However, the accuracy increases to $94 \%$ when the decision is taken using the weighting vote. Also, the task of gender identification is solved by texture and geometric features, which can be determined by local binary pattern (LBP) and gray level cooccurrence matrices (GLCM) [59]. More recently, several enhanced versions of LBP are developed for face and gender recognition as local directional pattern (LDP), local phase quantization (LPQ). In the same context, a novel variant of LBP is proposed by [60] named Adaptive patch-weight LBP (APWLBP). Their method used a pyramid structure to compute the gradient using weight parameters determined by Eigen theory. The main objective of (APWLBP) is to determine the optimal projection on the hyper-plane with a high value of variance for gender recognition. The performance of APWLBP based SVM is very competitive against CNN on three-dimensional Adience and LFW datasets. In the same context, Scale-invariant feature transform (SIFT) is combined with trainable features (CROSSFIRE) [61].

\subsection{Deep-learned features}

A novel synergy between CNN and ELM is illustrated by the work of [62] for age and gender identification. The CNN is used as an extractor of features, while ELM is used as a classifier to simultaneously determine the person's age and distinguish between male and female from face images.

63] employed two architectures of pretrained-CNN named VGG16 and ResNet for measuring ethnicity and gender informations.

64] designed four architecture of pretrained CNN: AlexNet, VGG-16, ResNet-152 and WideResNet-16-8 for predicting the age and gender over IMDB-WIKI datasets. The experiment study 
showed that Wide-ResNet presented a high performance in accuracy compared to others pre-trained CNN.

65] explored human facial analysis using three points: Face recognition, Gender and expression recognition. This study focuses only on gender recognition, so the authors used CNN for gender recognition.

66 used CNN as extractor for predicting age and gender from facial images. Then, the reduction of dimensionality is applied using PCA, and the task of classification is implemented using Feed-Forward Neural Network.

67] proposed a specific deep CNN architecture for real time gender identification using smartphones. The architecture comprises 4 convolutional layers, three max-pooling layers, 2 fully connected layers, and a single layer for regression. The training is realized by fusing two datasets FEI and CAS-PEAL, including 200 persons with 2800 faces and 1040 persons with 30,871. For the FEI dataset, the Deep-gender registered $98.75 \%$ as accuracy by considering a specific process including alignment before reducing the size of the facial image. However, the proposed method reached $97.73 \%$ in terms of accuracy for CAS-PEAL-R1 datasets. It is important to indicate that the authors split thier dataset to 5 fold as cross- validation.

\subsection{Deep-learned assisted by handcrafted features}

A significant number of study has been investigated about deep-learned features and their impact compared to handcrafted features and fused features (handcrafted with deep features) for gender identification [68].

The work of 69 summarized firstly, some methods based on handcrafted as LBP, HOG, SIFT, weighted HOG, and CROSSFIRE Filter and secondly, the authors explained the role of CNN which can be used for double tasks, i.e., used as an extractor of features and classifier in order to recognize age and gender from the face.

In [70], the authors have employed three methods including LBP, HOG and PCA as handcrafted features, deep CNN features and fused features based on three combinations named LBP-DL, HOGDLand PCA-DL. Furthermore, the task of gender identification is realized by two classifiers SVM and CNN. The experimental results shown a high rate of average accuracy $88.1 \%$ obtained by fused features (LBP-DL) and SVM classifier, tested on two datasets LFW and Adience. Additionally, [71] designed three occlusion methods assisted by AdienceNet and VGG16 for recognizing age and gender tasks.

\section{Features extraction}

In this section, several methods have been proposed to describe the texture characteristic. We present below a brief study of some existing techniques about the extraction of texture features, which applied to the analysis of facial images. The purpose of extracting descriptors (characteristic) in pattern recognition is to express primitives in a numerical or symbolic form called encoding. In this part, we will introduce the descriptors used in the experiments and results part. These are first of all the local binary patterns (LBP), then the descriptors of histogram based on oriented gradient (HOG), and finally the gray-level co-occurrence matrix (GLCM).

\subsection{Local binary patterns (LBP)}

Texture descriptors based on local binary patterns were initially proposed by [72]. The computation of the resulting image from the LBP application is akin to a correlation operation while applying a filter to a digital image. It suffices to process each pixel of the image by considering 
the eight pixels of its immediate neighborhood. The neighborhood of a pixel forms a matrix of $3 \times 3$ pixels where the pixel to be processed is in the center, and its neighborhood is around. Fig. 1 shows an example of the execution of the LBP algorithm relating to the steps described below.

Step 1 - Extraction of the neighborhood of the pixel to be processed. The eight intensity values of pixel's neighborhood to be processed are extracted from a matrix of $3 \times 3$ pixels. In this example, each pixel has a different gray intensity value. The pixel being processed has the value of intensity 40 .

Step 2 - A thresholding is performed on the intensity value of the neighboring pixels. Any pixel having an intensity value greater than or equal to the intensity value of the pixel being processed is assigned the value 1 . The value 0 is assigned to any intensity value lower than that of the pixel being processed.

Step 3 -A multiplier matrix is stored. This matrix will be used to describe the resulting local binary form uniquely in the next step of the algorithm.

Step 4 -Element-by-element multiplication. This operation is carried out between the matrix resulting from the thresholding of step 2 and the multiplying matrix of step 3.

Step 5 -The summation of the values of the resulting matrix from step 4 is performed. This sum is related in the output image to the corresponding coordinates of the pixel to be processed in the input image. The algorithm re-executes steps 1 to 5 until all the pixels of the input image are processed. According to the the procedure for identifying LBP, a histogram is calculated to characterize the frequency of appearance of the various patterns. The computed number for each pixel in step 5 uniquely identifies a gray intensity pattern among the possible patterns. The shape of the resulting histogram is characteristic of the texture studied by the LBP algorithm.

In general, the task of extracting features from facial images using LBP starts by dividing the input image into several blocks $(7 \times 7)$. Then, we extract the histogram for each block based on LBP. The final step consists of concatenating all histograms in order to realize the task of gender recognition. The concept of handcrafted features using LBP is shown in Fig. 2. To calculate the LBP code in a neighborhood of $P$ pixels with a radius $R$, we simply count the occurrences of gray levels $g_{p}$ greater than or equal to the central value using Eq.(1.

$$
L B P_{P, R}\left(x_{c}, y_{c}\right)=\sum_{p=1}^{P} s\left(g_{p}-g_{c}\right) 2^{p-1}
$$

Where $g_{p}$ and $g_{c}$ are the gray levels of a neighboring pixel and of the central pixel, respectively. $S$ indicates the Heaviside function defined by Eq. 2

$$
S(x)=\left\{\begin{array}{c}
+1 \text { if } \quad x \geq 0 \\
0 \text { if } \quad x<0
\end{array}\right.
$$

\subsection{Histogram of Oriented Gradient (HOG)}

HOG is a very powerful descriptor proposed by Dalal and Triggs in 2005, which was initially developed for human detection [73]. However, later it is extended and applied to other topics of computer vision problems including facial recognition [74], gender and age estimation [75], detection of plant pathology's [76] and recognition of facial expressions [77]. HOG describes the 

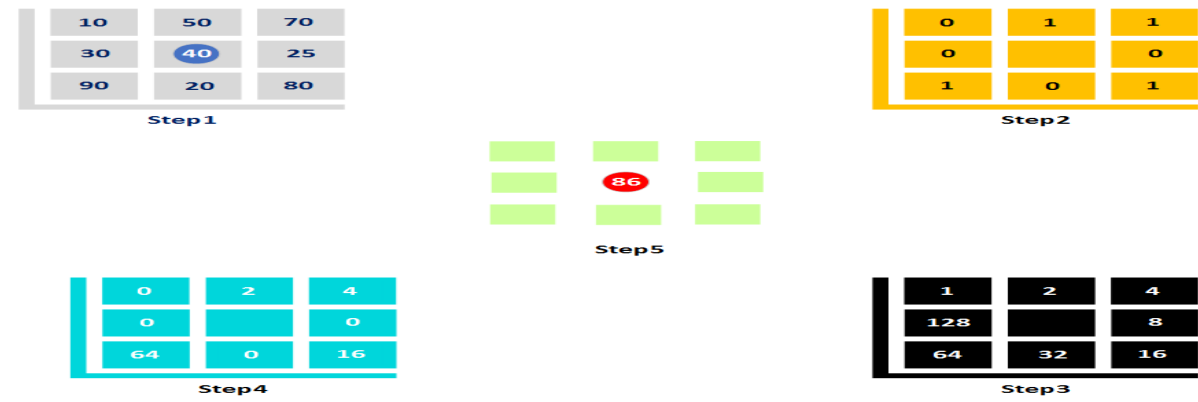

Figure 1: Basic LBP operator.

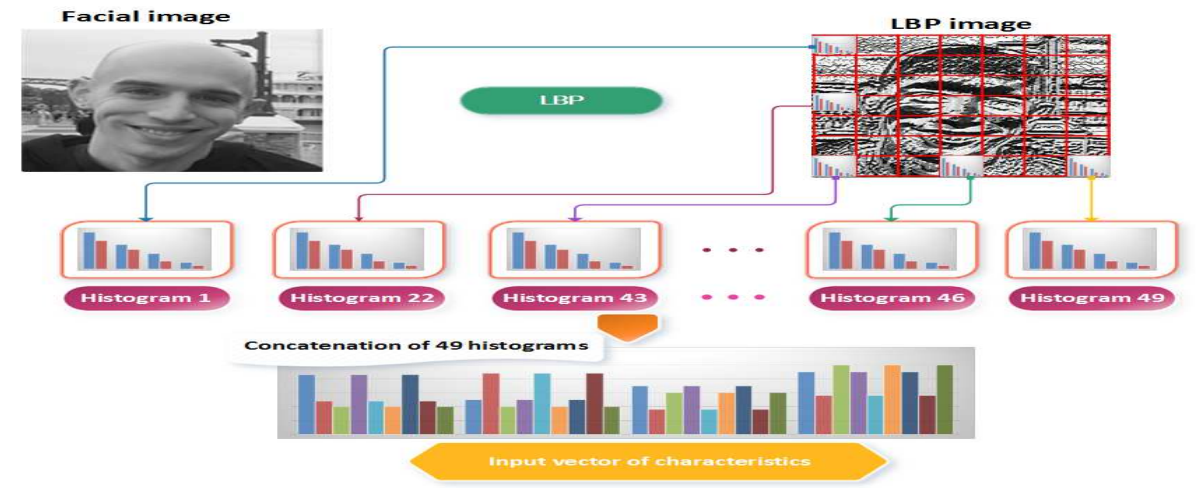

Figure 2: The coding face using a set of LBP histogram.

appearance and local shape of the object in an image using the distribution of gradients. The characteristic vector of an image $I(x, y)$ using the HOG technique is obtained by the following procedure:

Step 1 - Divide the image $I(x, y)$ into equal blocks $\left(N_{b} \times N_{b}\right)$, where each block contains $(M \times M)$ regular cells of size $(8 \times 8)$ pixels.

Gradient values $\left(G_{h}, G_{y}\right)$ are computed for each pixel using a centered $1-D$ derivative filter, in the horizontal and vertical directions. For this, the following masks $\left(S_{h}, S_{v}\right)$ are used and defined by Eq.(3) and Eq. (4):

$$
\begin{gathered}
S_{h}=\left[\begin{array}{lll}
-1 & 0 & 1
\end{array}\right] \\
S_{v}=\left[\begin{array}{c}
-1 \\
0 \\
1
\end{array}\right] \\
G_{h}(x, y)=I(x, y) * S_{h} \\
G_{v}(x, y)=I(x, y) * S_{v}
\end{gathered}
$$

Step 2 - The magnitude $(|G(x, y)|)$ and gradient orientation $(\theta)$ of each pixel $(x, y)$ are calculated using Eq. (7) and Eq.(8):

$$
\begin{gathered}
|G(x, y)|=\sqrt{G_{h}^{2}(x, y)+G_{v}^{2}(x, y)} \\
\theta=\arctan \left(\frac{G_{v}(x, y)}{G_{h}(x, y)}\right)
\end{gathered}
$$


$G_{h}$ and $G_{v}$ represent the horizontal gradient and the vertical gradient at pixel (x, y), respectively.

Step 3 - The histogram of the orientation based on the gradient inside each cell is calculated by quantizing unsigned gradients at each pixel in 9 channels (bins) orientations. The histograms are uniform from 0 to $180^{\circ}$ (unsigned case) or from 0 to $360^{\circ}$ (signed case).

Step 4 - The characteristic vector for each cell is normalized using histograms in their recognized blocks. In this work we use the L2-norm for the normalization of the blocks; the normalization factor is calculated using the following equation:

$$
\text { Hist }_{n}=\frac{\text { Hist }}{\sqrt{\|H i s t\|_{2}^{2}+\epsilon}}
$$

Where Hist is the non-normalized vector containing all the histograms in a block, $\|H i s t\|_{2}$ is the L2 norm of the descriptor vector, and $\epsilon$ is a regularization term.

Step 5 - The characteristic vector of each block is formed by concatenating the histogram vectors of all the cells in the block. The characteristic vector HOG is formed by concatenating the characteristic vectors of all the blocks for a given image.

\subsection{Grey level co-occurrence matrix (GLCM))}

GLCM is a method widely used in the field of image processing which belongs to the class of texture-based statistical methods. Textural content is expressed differently depending on distance $d$ and orientation $\theta$ of the displacement considered between the pairs of sites, which provides 4 GLCMs due to the 4 orientations defined by : $\theta_{1}=0^{\circ}, \theta_{2}=45^{\circ}, \theta_{3}=90^{\circ}$ and $\theta_{4}=135^{\circ}$ [78]. Fig. 3 ] illustrates the configuration of 4 GLCMs that correspond to the 4 directions with the distance fixed to $d=1$. The extracted features from GLCMs include mainly the energy, the contrast, entropy, correlation, homogeneity, dissimilarity, the cluster shade and the cluster prominence, which are explained succinctly by:

- The energy $\left(E_{n}\right): E_{n}$ expresses the regularity of the texture, which can be computed by:

$$
E_{n}=\sum_{l=0}^{L-1} \sum_{m=0}^{L-1} P_{(l, m)}^{2}
$$

It is important to note that a higher value of $\left(E_{n}\right)$ signify a complete homogeneous image.

- The contrast $\left(C_{n}\right)$ : It measures the rate of local variation in the picture $(I)$. The formula of $\left(C_{n}\right)$ is given by :

$$
C_{n}=\sum_{l=0}^{L-1} \sum_{m=0}^{L-1} P_{(l, m)}|l-m|^{2}
$$

- The entropy $\left(E_{t}\right): E_{t}$ is the inverse of energy and characterizes the irregular appearance of the image, hence a strong correlation between these two attributes. The formula of $E_{t}$ is computed by :

$$
E_{t}=\frac{1}{2 \log (N)} \sum_{l=0}^{L-1} \sum_{m=0}^{L-1} P_{(l, m)} \log _{2} P_{(l, m)}
$$

- Correlation $\left(C_{r}\right)$ : It can be compared to a measure of the linear dependence of gray levels in the image. It calculated by: 


$$
C_{r}=\sum_{l=0}^{L-1} \sum_{m=0}^{L-1} \frac{\left(l-\mu_{l}\right)\left(m-\mu_{m}\right)}{\sigma_{l} \sigma_{m}} P_{(l, m)}
$$

- Homogeneity $\left(H_{m}\right)$ :The homogeneity changes inversely to the contrast and takes on high values if the differences between the analyzed pixel pairs are weak. It is therefore more sensitive to the elements diagonals of the GLCM, unlike the contrast which depends more on the distant elements diagonal. It is measured by:

$$
H_{m}=\sum_{l=0}^{L-1} \sum_{m=0}^{L-1} \frac{P_{(l, m)}}{1+|l-m|^{2}}
$$

- Dissimilarity $\left(D_{s}\right)$ : It expresses the same characteristics of the image as contrast to difference that the weight of the GLCM inputs increases linearly as they move away from the diagonal rather than quadratically in the case of contrast.

It calculated by:

$$
D_{s}=\frac{1}{(L-1)^{2}} \sum_{l=0}^{L-1} \sum_{m=0}^{L-1} P_{(l, m)}|l-m|
$$

- The cluster shade and the cluster prominence give information on the degree of symmetry of the GLCM.

- The cluster shade is defined by :

$$
C_{s}=\sum_{l=0}^{L-1} \sum_{m=0}^{L-1} P_{(l, m)}\left(l-\mu_{l}+m-\mu_{m}\right)^{3}
$$

whereas, the cluster prominence is given by:

$$
C_{p}=\sum_{l=0}^{L-1} \sum_{m=0}^{L-1} P_{(l, m)}\left(l-\mu_{l}+m-\mu_{m}\right)^{4}
$$

Similar to Multi-blocks LBP/HOG, the whole image is divided into $7 \times 7$ blocks, where from each block, the statistical moments GLCM are extracted and then combined together to generate the descriptors vectors.

\section{Archimedes optimization algorithm (AOA)}

AOA is an algorithm inspired by physics more particularly Archimedes' law. This algorithm is introduced by Fatma Hashim in 2020 and belongs to the class of meta-heuristics [37]. The particularity of this algorithm lies in the encoding of the solution, which encompasses three auditory information: Volume $(V)$, Density $(D)$ and Acceleration $(\Gamma)$ to the basic agents. So, initially the group of agents is generated randomly in Dim dimensions. As additive data, random values of $V$, $D$ and, $\Gamma$ are provided. After, the evaluation process is realized for each object to determine the best object $\left(O_{b e s t}\right)$. 


\section{Input data (Image)}

\begin{tabular}{|c|c|c|c|c|c|c|c|c|}
\hline & \multicolumn{5}{|c|}{$r \cdots-\cdots-\cdots-\cdots-\cdots-$} \\
\hline 2 & 2 & 1 & 1 & $i$ & 4 & 0 & 1 & 1 \\
\hline 2 & 2 & 1 & 1 & 4 & 2 & 2 & 0 & 0 \\
\hline 1 & 1 & 3 & 3 & $\vdots$ & 0 & 0 & 1 & 0 \\
\hline 1 & 1 & 4 & 2 & 1 & 0 & 1 & 0 & 0 \\
\hline
\end{tabular}

\begin{tabular}{|c|c|c|c|c|c|c|c|}
\hline 2 & 2 & 1 & 1 \\
\hline 2 & 2 & 1 & 1 \\
\hline 1 & 1 & 3 & 3 & 4 & 2 & 0 & 0 \\
\hline 1 & 1 & 4 & 2 & 0 & 2 & 1 & 0 \\
\hline $\operatorname{GLCM}\left(\theta=90^{\circ} ; d=1\right)$ & $\vdots$ & 0 & 0 & 0 \\
\cline { 3 - 9 } & & 0 & 0 & 1 & 0 \\
\hline
\end{tabular}

\begin{tabular}{c|c|c|c|}
\hline 2 & 2 & 1 & 1 \\
\hline 2 & 2 & 1 & 1 \\
\hline 1 & 1 & 3 & 3 \\
\hline 1 & 1 & 4 & 2 \\
\hline $\operatorname{GLCM}\left(\theta=45^{\circ} ; d=1\right)$ \\
$\vdots$ \\
\hline
\end{tabular}

\begin{tabular}{|l|l|l|l|}
\hline 3 & 1 & 1 & 0 \\
\hline 1 & 1 & 0 & 0 \\
\hline 1 & 0 & 0 & 0 \\
\hline 0 & 0 & 1 & 0 \\
\hline
\end{tabular}

\begin{tabular}{|l|l|l|l|}
\hline 2 & 2 & 1 & 1 \\
\hline 2 & 2 & 1 & 1 \\
\hline 1 & 1 & 3 & 3 \\
\hline 1 & 1 & 4 & 2 \\
\hline
\end{tabular}

\begin{tabular}{|l|l|l|l|}
\hline 2 & 2 & 0 & 0 \\
\hline 0 & 1 & 1 & 0 \\
\hline 1 & 1 & 0 & 0 \\
\hline 1 & 0 & 0 & 0 \\
\hline
\end{tabular}

Figure 3: An example of GLCMs based on different orientations. 
During the process of AOA, the update of density and volume is realized in order to change the acceleration based on the collision concept between objects, which play an important role to determine the novel position of current solution. The general steps of AOA are described as following:

-The first step - Initialization:. This step aims to initialize randomly the real population that contains $N$ objects using Eq. (18). Also, each object is characterized by their density $\left(D_{i}\right)$, volume $\left(V_{i}\right)$ and acceleration $\left(\Gamma_{i}\right)$ which are defined in random way using the following equations Eq. (19), Eq. 200 and Eq. (21):

$$
\begin{gathered}
O_{i}=O_{i}^{M i n}+r_{1} \times\left(O_{i}^{M a x}-O_{i}^{M i n}\right) ; i=1,2, \ldots, N \\
D_{i}=r_{2} \\
V_{i}=r_{3} \\
\Gamma_{i}=\Gamma_{i}^{M a x}+r_{4} \times\left(\Gamma_{i}^{M a x}-\Gamma_{i}^{M i n}\right) ; i=1,2, \ldots, N
\end{gathered}
$$

Where $O_{i}$ represents the $i^{t h}$ object, $O_{i}^{M a x}$ and $O_{i}^{\text {Min }}$ are the maximal and minimal limits of the search-space, respectively.

$r_{1}, r_{2}, r_{3}$ and $r_{4}$ are a random vectors which belong to $[0,1]^{\text {Dim }}$.

The population will be evaluated by computing the score for each object in order to determine the best object $\left(O_{B e s t}\right)$ by joining their best values of density $\left(D_{\text {Best }}\right)$, volume $\left(V_{\text {Best }}\right)$ and acceleration $\left(\Gamma_{\text {Best }}\right)$.

- The second step - The update of densities $\mathcal{E}$ volumes. In this step, the values of density and volume for each object are updated by the control of the best density and best volume using Eq. 22 and Eq. (23):

$$
\begin{gathered}
D_{i}^{t+1}=D^{t}+s_{1} \times\left(D_{\text {Best }}-D_{i}^{t}\right) \\
V_{i}^{t+1}=V_{i}^{t}+s_{2} \times\left(V_{\text {Best }}-V_{i}^{t}\right)
\end{gathered}
$$

Where $s_{1}, s_{2}$ are random scalars in $[0,1]$.

- The third step - Transfer coefficient $\mathbb{E}$ density scalar:. In this step the collision between object is occurred until obtaining the equilibrium state. The principal role of transfer function $\left(T_{c}\right)$ is to switch from exploration to exploitation mode, defined by Eq. (24):

$$
T_{c}=\exp \left(\frac{t-T}{T}\right)
$$

The $T_{c}$ increases exponentially over time until reaching $1 . t$ is the current iteration, while $T$ denotes the maximum number of iterations. Also, the decrease of density scalar $d_{s}$ in AOA allows to find an optimal solution using Eq. 25]:

$$
d_{s}^{t+1}=\exp \left(\frac{t-T}{T}\right)-\left(\frac{t}{T}\right)
$$

- The fourth step - Exploration phase : In this step, the collision between agents is occurred using a random selection of material $(M r)$. So, the update of acceleration objects is applied using Eq. 267 when the transfer function value is less or equal to 0.5 . 


$$
\Gamma_{i}^{t+1}=\frac{D_{M r}+V_{M r} \times \Gamma_{M r}}{D_{i}^{t+1} \times V_{i}^{t+1}}
$$

- The fifth step - Exploitation phase : In this step, the collision between agents is not realized. So, the update of acceleration objects is applied using Eq. (27) when the transfer coefficient value is greater than 0.5 .

$$
\Gamma_{i}^{t+1}=\frac{D_{\text {Best }}+V_{\text {Best }} \times \Gamma_{\text {Best }}}{D_{i}^{t+1} \times V_{i}^{t+1}}
$$

Where $\Gamma_{\text {Best }}$ is the acceleration of the optimal object $O_{B e s t}$.

- The sixth step - Normalization of acceleration $\therefore$. In this step, we normalize the acceleration in order to determine the rate of change using (28):

$$
\Gamma_{i-\text { norm }}^{t+1}=\alpha \times \frac{\Gamma_{i}^{t+1}-\Gamma^{M i n}}{\Gamma^{M a x}-\Gamma^{M i n}}+\beta
$$

Where $\alpha$ and $\beta$ are fixed to 0.9 and 0.1 , respectively. The $\Gamma_{i-\text { norm }}^{t+1}$ determines the percentage of step that each agent will change. The higher value of acceleration means that the object realizes the operation of exploration; otherwise, the exploitation mode is operational.

- The seventh step - The Update process: For exploration phase $\left(T_{c} \leq 0.5\right)$, the position of $i^{\text {th }}$ object in iteration $t+1$ is modified by Eq. (29), whereas the object position is updated by Eq. (30) in exploitation phase $\left(T_{c}>0.5\right)$.

$$
O_{i}^{t+1}=O_{i}^{t}+c_{1} \times r_{5} \times \Gamma_{i-n o r m}^{t+1} \times d_{s} \times\left(O_{\text {rand }}-O_{i}^{t}\right)
$$

Where $c_{1}$ is equal to to 2 .

$$
O_{i}^{t+1}=O_{B e s t}^{t}+F \times c_{2} \times r_{6} \times \Gamma_{i-n o r m}^{t+1} \times d_{s} \times\left(\delta \times O_{B e s t}-O_{i}^{t}\right)
$$

where $c_{2}$ is fixed to 6 .

The parameter $\delta$ is positively correlated with the time and this parameter is proportionally linked to the transfer coefficient $T_{c}$ i.e $\delta=2 \times T_{c}$. The main role of this parameter is to ensure a good balance between exploration and exploitation operations. During the first iterations, the margin between the best object and the other object is higher, which provides a high random walk. However, in last iterations, the margin will be reduced and provided a low random walk.

$F$ is employed for flagging which controls search direction using Eq.(31):

$$
F=\left\{\begin{array}{lll}
+1 & \text { if } \quad \zeta \leq 0.5 \\
-1 & \text { if } \quad \zeta>0.5
\end{array}\right.
$$

where $\zeta=2 \times$ rand -0.5 .

- The eighth step - The evaluation:. In this step, we evaluate the novel population using score index $S c$ in order to determine the best object $O_{B e s t}$ and the best additive information including $D_{\text {Best }}, V_{\text {Best }}$, and $\Gamma_{\text {Best }}$. 


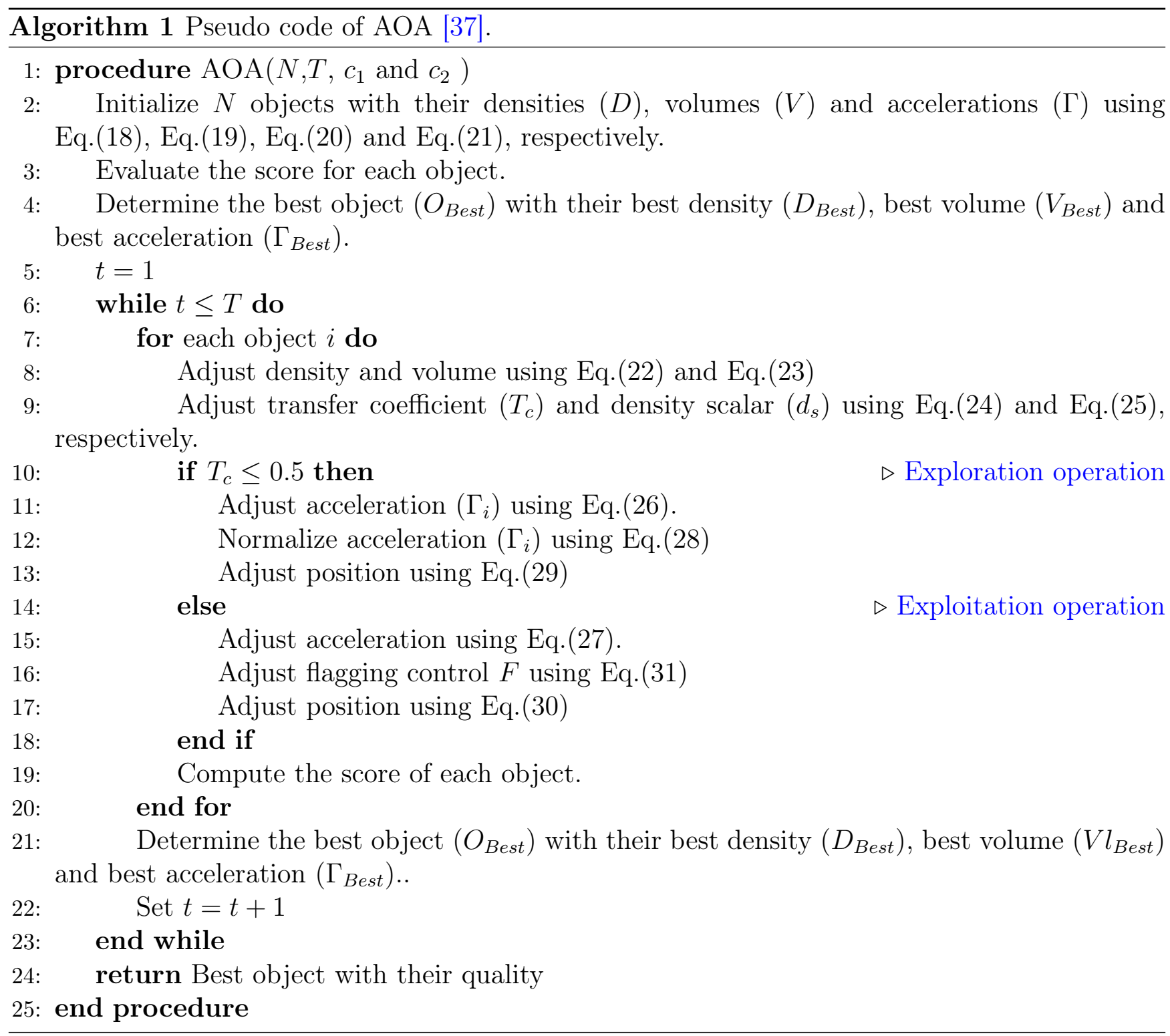




\section{AOA based FS for Gender Recognition}

This section explains our system of gender recognition using Archimedes optimizer algorithm (AOA) based feature selection. This system required three key points:

- The encoding solution;

- The evaluation of score;

- The architecture system of gender recognition

\subsection{Structure of immersed object}

This ingredient plays a vital role in optimization process using physical or swarm algorithms.

The size of each object in AOA corresponds to the number of blocks, which is a 1-D vector with 49 elements. The components of the vector are randomly generated in the range $[0,1$. At this stage, if the value is greater or equal to 0.5 , thus the value is rounded to one. In this case, the bloc is considered as a relevant feature and coded by a histogram of (LBP, HOG, or GLCM). Contrarily, the block is ignored when the value is rounded to zero. This vector will be transformed to a matrix with a size of $7 \times 7$ in order to project the encoding solution on the image for extracting the multi -blocks-based-histograms.

This encoding aims to select the informative areas by realizing the concatenation of activated Multi-blocks based histograms (LBP, HOG, or GLCM). The encoding object is shown in Fig 4. According to this Figure, we can see clearly that the current object selects fifteen blocks based histograms from 49 face areas, which will be concatenated and served as the input of the neural network classifier.

\subsection{Score evaluation}

In order to apply the process of gender recognition using a wrapper feature selection assisted by AOA, a good compromise between accuracy and a lower number of features must be assured. $\mathrm{SO}$, the score for each object is computed by:

$$
S c=0.99 \times A c c+0.01 \times\left(\frac{D-d}{D}\right)
$$

where $(A c c),(d)$ are the accuracy obtained by Multilayer perceptron neural network (MLP) and the size of selected histograms, respectively.

In Eq. (32), $D$ is the total number of multi-blocs based histogram extracted from original image.

The MLP is integrated as a classifier in the FS process using $k$-folds as a cross-validation strategy. In this study, the value of $k$ is fixed to 5 to realize a fair comparison. So, $80 \%$ of samples is used in the training step, where the rest is used for testing. Additionally, the architecture of MLP is described in Fig. 5 .

This architecture includes three layers:

- Input layer: It corresponds to the multi-blocks-based histograms (LBP/HOG) input features or GLCM vector. So the number of neurons in this layer for LBP, HOG and GLCM are equal to $\left(\right.$ Blocks $_{\text {Selected }} \times 59$ bins $),\left(\right.$ Blocks $_{\text {Selected }} \times 9$ bins $)$ and $\left(\right.$ Blokcs $\left._{\text {Selected }} \times 8\right)$, respectively.

- Hidden layer: It contains the double of neurons used in the input layer.

- Output layer: It contains two neurons; the first one corresponds to male, while the second is reserved for female.

It is important to indicate that the higher value of the computed score through all objects is assigned to the best object $\left(O_{B e s t}\right)$. 


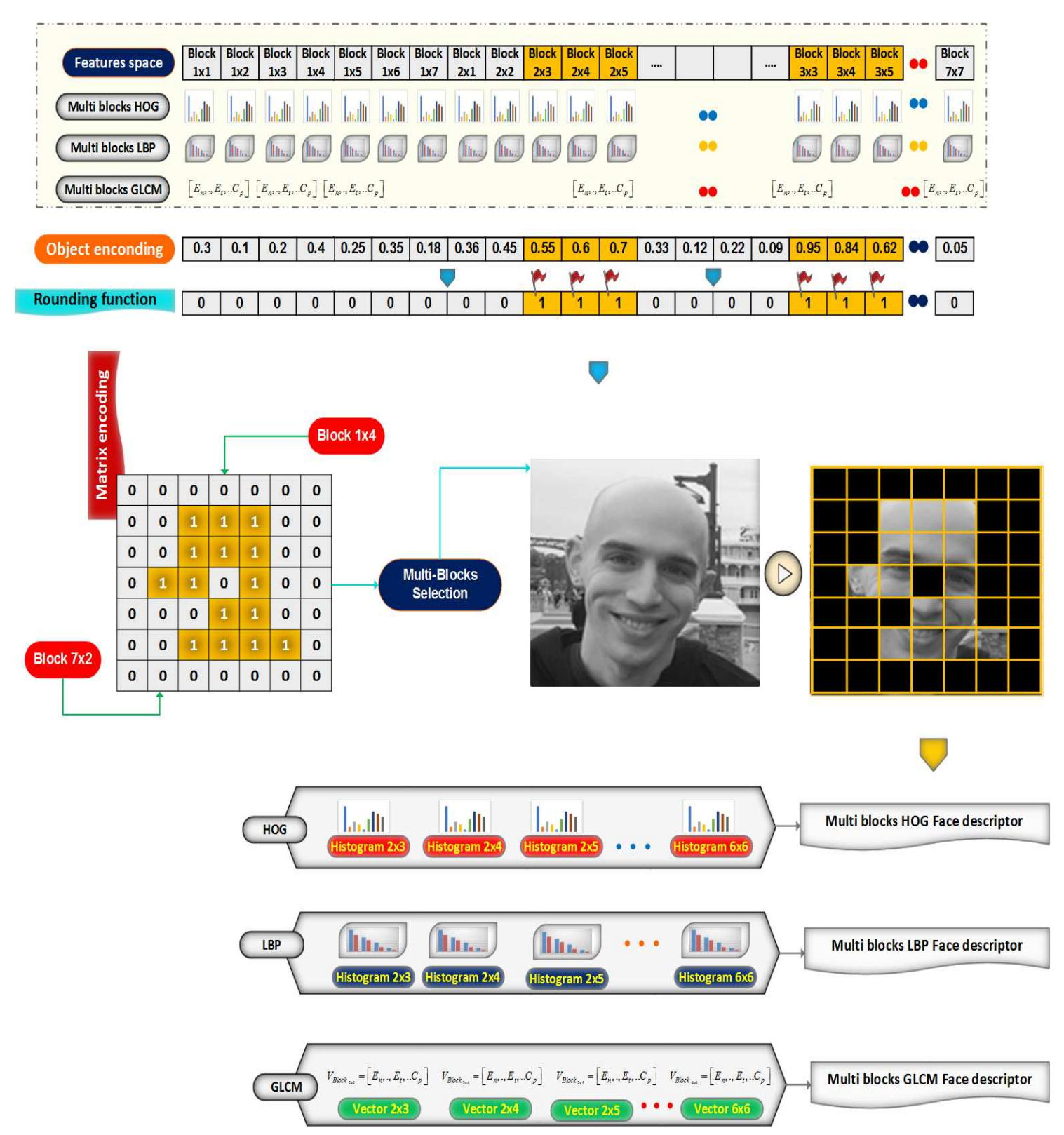

Figure 4: Encoding solution.

\subsection{Design framework}

This part represents the core of our work, which consists of applying the AOA algorithm in gender recognition based on the selection of attributes. To better understand the proposed architecture, we preferred to explain the essential ingredients in bullet points:

- Initialization: This step is started by creating a random population of $N$ immersed objects with $7 \times 7$ as dimension (49) elements.

- Encoding solution: This step aims to transform random objects into binary vectors to select the relevant blocks-based histograms or GLCM features.

- Selection of subset features: After decoding the object as illustrated in Fig. 4, the corresponding blocks are determined from the datasets based on LBP, HOG, or GLCM. 


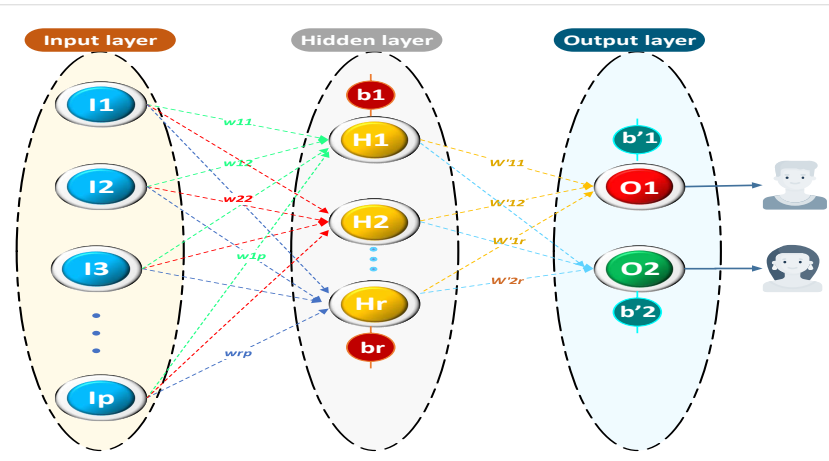

Figure 5: The architecture of MLP.

- Score evaluating: For each object generated by AOA that indicates the sub-set of selected features must be assessed using MLP classifier. The score represents the relationship between accuracy and the selected number of features, computed by Eq. 32 .

- The update of position: The most important step in our architecture consists of applying a sequence of operators as updating densities and volumes, exploring/ exploiting tasks, and norming of acceleration in order to produce better solutions with a higher score as shown in Alg. 1.

- Stop condition: The cycle of AOA is an iterative process, controlled by the maximum number of iterations as a stop condition.

It is important to indicate that the MLP is performed using 5 -folds cross-validation, which means that the MLP is trained 5 times and the average evaluation of fitness is computed. Figure. 6 illustrates the overall steps of the AOA-based gender recognition and FS process.

\section{Experimental results}

In order to realize a fair analysis, the efficiency of AOA is compared with different and recent computational algorithms inspired from swarm intelligence, mathematical algorithm and physical algorithms including HHO, MRFO, EPO, SCA, EO, HGSO and MVO, tested on two datasets (GT \& FEI datasets) in the same condition by employing three textural desciptors like HOG, LBP and GLCM.

\subsection{Simulation setup}

\subsubsection{Statistical metrics}

In order to investigate the efficiency of the AOA algorithm in the field of facial analysis based FS, especially in gender recognition.

The confusion matrix must be used and defined in Table 1. Next, some measures must be computed as Accuracy $(A c)$, Recall $(R e)$, Precision $(P r)$ and F-score $\left(F_{\text {score }}\right)$.

Where : - TrP: The classifier identifies correctly the person knowing that their class is male;

- TrN: the classifier identifies the person correctly knowing that their class is female;

- FaP: the classifier assigns the person to the male class knowing that the example belongs to the female class

- FaN: the classifier assigns the person to the female class knowing that the example belongs to the male class. 


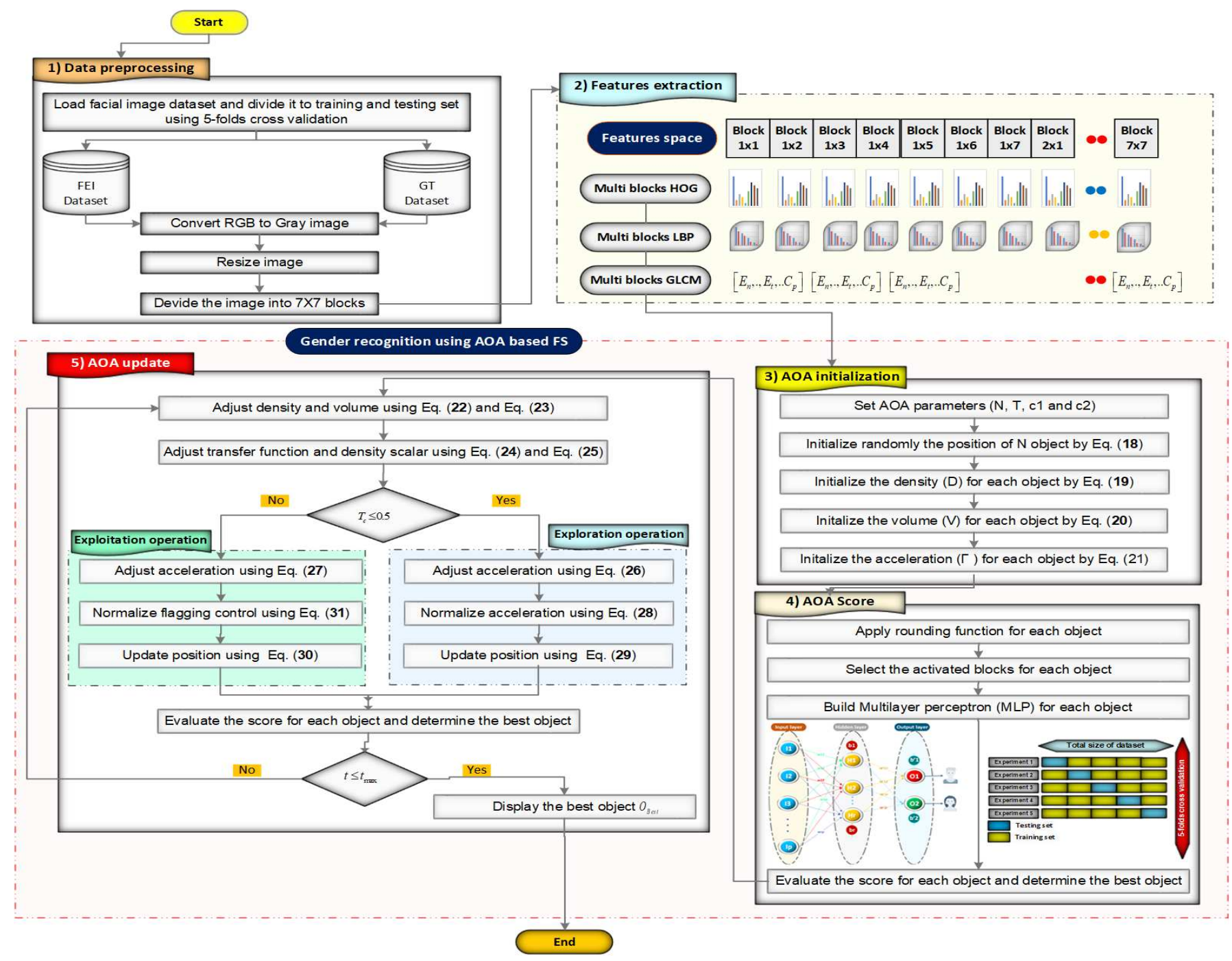

Figure 6: The design framework of AOA based FS for gender recognition.

- The accuracy metric $(A)$ : Among the most important metrics, we find the accuracy which measures tHE rate of correct data classification, defined by :

$$
A=\frac{\operatorname{Tr} P+\operatorname{Tr} N}{\operatorname{Tr} P+F a N+F a P+\operatorname{Tr} N}
$$

- The recall metric $(R)$ : This metric is called also true positive rate (TPR), which indicates the percentage of predicting male person as:

$$
R=\frac{\operatorname{Tr} P}{\operatorname{Tr} P+F a N}
$$

- The precision $(P)$ : It indicates the rate of true predicted samples as:

$$
P=\frac{\operatorname{Tr} P}{F a P+\operatorname{Tr} P}
$$


Table 1

Confusion matrix.

\begin{tabular}{|c|c|c|}
\cline { 2 - 3 } \multicolumn{1}{c|}{} & \multicolumn{2}{c|}{ Predicted } \\
\hline Actual & Male & Female \\
\hline Male & TrP & FaN \\
\hline Female & FaP & TrN \\
\hline
\end{tabular}

\footnotetext{
${ }^{1}$ https://fei.edu.br/ cet/facedatabase.html

${ }^{2}$ http://www.anefian.com/research/face eco.htm $_{r}$
} recognition. cision. It computed by Eq 37 :

\subsubsection{Parameters settings}

\subsection{Description of datasets} resolution of each image is $640 \times 480$.

$$
S_{r}=\frac{d}{D}
$$

where $d$ is the number of relevant blocks based faces which increase the performance of gender

- F-score $\left(F_{\text {Score }}\right)$ : In statistical F-score indicates the harmonic mean between recall and pre-

$$
F_{S c}=2 \times \frac{R \times P}{R+P}
$$

- $\mathrm{CPU}$ time $(\mathrm{Cpu})$ : It is the required time for each algorithms.

This sub-section defines all parameters used for each optimizer. It is important to enumerate the list of algorithms used for realizing the task of gender recognition from faces. The parameters settings of HHO, MRFO, EPO, SCA, EO, HGSO and MVO are defined in Table 2.

- FEI dataset: It is a Brazilian dataset that contains 200 individuals. It is important to note that each individual has 14 images thus a total of 2800. The images were captured on a white background and of color quality. The age category is between 19 and 40 years old. Some changes in the appearance of the face such as hairstyle and adornments have also been incorporated. The dataset is balanced because half of the examples are men and half are women 1 . We note that the

- Georgia Tech Face dataset (GT) : It contains 50 people captured in two sessions between 04/06/99 and 11/15/99. Each person contains 15 images so a total of 750 images of size $640 \times 480$ pixels. The average size of a face is $150 \times 150$ pixels. Images are frontal expressed in conditions of light, change of scale and expression. This dataset contains 7 women and 43 men 2 . 
Table 2

Parameters settings of physical, mathematical and swarm inspired algorithms.

\begin{tabular}{|c|c|}
\hline Algorithms & Parameters setting \\
\hline Common settings & $\begin{array}{l}\text { Population size }(N=10) \\
\text { Maximum number of iterations }(T=100) \\
\text { Maximal limit }=1 \\
\text { Minimal limit }=0 \\
\text { Dimension corresponds to the number of blocks }(D=49)\end{array}$ \\
\hline $\mathrm{AOA}$ & $\begin{array}{l}c_{1}=2 \text { and } c_{2}=6 \\
\alpha=0.9 \text { and } \beta=0.1\end{array}$ \\
\hline $\mathrm{EO}$ & $\begin{array}{l}a_{1}=2 \\
a_{2}=1 \\
\text { Generation Probability }(G P=0.5)\end{array}$ \\
\hline MVO & $\begin{array}{l}\text { Wormehole Existance Prob } \\
\left(W E P_{\max }=1 \text { and } W E P_{\min }=0.5\right) \\
\text { Traveling Distance Rate }(T D R \in[0.5 ; 1])\end{array}$ \\
\hline HGSO & $\begin{array}{l}\text { Clusters number }=2 \\
M_{1}=0.1 \text { and } M_{2}=0.2 \\
\alpha=\beta=1 \text { and } K=1 \\
l_{1}=5 E-03, l_{2}=1 E+02 \text { and } l_{3}=1 E-02\end{array}$ \\
\hline EPO & $\begin{array}{l}\text { Temperature Profile }\left(T^{\prime} \in[1 ; 1000]\right) \\
A \in[-1,1.5] \\
M=2 \\
f \in[2,3] \\
l \in[1.5,2] \\
\text { Function } S() \in[0,1.5]\end{array}$ \\
\hline $\mathrm{SCA}$ & $\mathrm{a} \in[2,0]$ \\
\hline MRFO & $S=2$ \\
\hline $\mathrm{HHO}$ & $\begin{array}{l}N=10 \\
T=100 \\
D \text { indicates to features number } \\
\beta=1.5\end{array}$ \\
\hline
\end{tabular}




\subsection{Results \& Discussion}

\section{- In terms of fitness and Cpu time :}

Table. 3 summarizes the results of the AoA algorithm based onwrapper FS in terms of fitness by varying the extraction methods (HOG, LBP \& GLCM), tested on two GT \& FEI databases for achieving the task of gender recognition.

By analyzing the results of Table. 3, we first notice a precise performance obtained by the AOA algorithm based on the three extraction methods for the two GT \& FEI databases. Second, AOA-based HOG achieves a higher value of of fitness than AOA-based on LBP and GLCM for both datasets.

Furthermore, it can be observed a great competitively between AOA-based HOG and AOAbased GLCM for both datsets. Also, it is essential to show that EO is ranked second place for both datasets.

The time consumed by the optimization methods based on wrapper Fs varying the extraction methods is shown in Table. 4. The SCA algorithm is the fastest for the FEI database compared to other competitors based on the three descriptors (HOG, LBP \& GLCM). For the GT database, EO requires less time based on LBP and GLCM compared to other optimizers. Note also that the HOG-based EPO algorithm is ranked first in terms of execution time compared to other algorithms.

Table 3

The impact of features descriptors on the performance of AOA against other recent optimizers over Fitness measures

\begin{tabular}{cccccccc}
\hline Fitness & \multicolumn{3}{c}{ GT dataset } & & \multicolumn{3}{c}{ FEI dataset } \\
\cline { 2 - 4 } \cline { 6 - 7 } Algorithms & HOG & LBP & GLCM & & HOG & LBP & GLCM \\
\hline HHO & 0.8947 & 0.8644 & 0.8913 & & 0.9776 & 0.9262 & 0.9849 \\
SCA & 0.8914 & 0.8653 & 0.8873 & & 0.9820 & 0.9518 & 0.9841 \\
EO & 0.9002 & 0.8642 & 0.8927 & & 0.9853 & 0.9461 & 0.9829 \\
EPO & 0.8966 & 0.8658 & 0.8887 & & 0.9804 & 0.9351 & 0.9780 \\
MRFO & 0.8950 & 0.8593 & 0.8902 & & 0.9837 & 0.9412 & 0.9853 \\
HGSO & 0.8902 & 0.8543 & 0.8833 & & 0.9837 & 0.9298 & 0.9834 \\
MVO & 0.8981 & 0.8589 & 0.8954 & & 0.9805 & 0.9335 & 0.9825 \\
AOA & $\mathbf{0 . 9 0 1 5}$ & $\mathbf{0 . 8 6 9 0}$ & $\mathbf{0 . 8 9 6 9}$ & & $\mathbf{0 . 9 8 8 2}$ & $\mathbf{0 . 9 5 3 4}$ & $\mathbf{0 . 9 8 5 8}$ \\
\hline
\end{tabular}

Table 4

The impact of features descriptors on the performance of AOA against other recent optimizers over Cpu Time measures

\begin{tabular}{ccccccccc}
\hline CPU time & \multicolumn{3}{c}{ GT dataset } & & \multicolumn{3}{c}{ FEI dataset } \\
\cline { 2 - 4 } \cline { 7 - 9 } Algorithms & HOG & LBP & GLCM & & HOG & LBP & GLCM \\
\hline HHO & 446.6000 & 466.0300 & 363.8200 & & 249.3200 & 295.8900 & 258.3600 \\
SCA & 301.8600 & 211.4300 & 264.7200 & & $\mathbf{1 8 1 . 5 4 0 0}$ & $\mathbf{1 1 8 . 8 4 0 0}$ & $\mathbf{1 4 4 . 1 0 0 0}$ \\
EO & 324.7700 & $\mathbf{1 8 2 . 8 2 0 0}$ & 221.4100 & & 250.8200 & 214.4500 & 295.8000 \\
EPO & $\mathbf{2 8 5 . 5 8 0 0}$ & 272.2800 & 324.0800 & & 248.1200 & 126.0400 & 211.3700 \\
MRFO & 301.8600 & 445.4700 & 468.4100 & & 302.8600 & 226.5200 & 218.7900 \\
HGSO & 454.8300 & 398.6100 & 312.1300 & & 282.4900 & 199.3700 & 229.3200 \\
MVO & 368.0900 & 430.3200 & 369.4800 & & 375.0500 & 272.1800 & 229.4900 \\
AOA & 391.3700 & 332.3200 & 388.8600 & & 197.0700 & 231.6700 & 340.9200 \\
\hline
\end{tabular}




\section{Table 5}

The impact of features descriptors on the performance of AOA against other recent optimizers over Accuracy measures

\begin{tabular}{ccccccccc}
\hline \multirow{2}{*}{$\begin{array}{c}\text { Accuracy } \\
\text { Algorithms }\end{array}$} & \multicolumn{3}{c}{ GT dataset } & & \multicolumn{3}{c}{ FEI dataset } \\
\cline { 2 - 4 } \cline { 7 - 9 } & HOG & LBP & GLCM & & HOG & LBP & GLCM \\
\hline HHO & 0.8990 & 0.8689 & 0.8960 & & 0.9831 & 0.9320 & 0.9887 \\
SCA & 0.8914 & 0.8660 & 0.8887 & & 0.9841 & 0.9534 & 0.9864 \\
EO & 0.9031 & 0.8651 & 0.8953 & & 0.9889 & 0.9506 & 0.9864 \\
EPO & 0.8993 & 0.8669 & 0.8917 & & 0.9840 & 0.9370 & 0.9815 \\
MRFO & 0.9028 & 0.8636 & 0.8944 & & 0.9893 & 0.9456 & 0.9889 \\
HGSO & 0.8944 & 0.8578 & 0.8873 & & 0.9881 & 0.9349 & 0.9886 \\
MVO & 0.9020 & 0.8624 & 0.8997 & & 0.9865 & 0.9392 & 0.9875 \\
AOA & $\mathbf{0 . 9 0 3 4}$ & $\mathbf{0 . 8 7 0 1}$ & $\mathbf{0 . 9 0 1 4}$ & & $\mathbf{0 . 9 9 1 6}$ & $\mathbf{0 . 9 5 6 1}$ & $\mathbf{0 . 9 9 0 4}$ \\
\hline
\end{tabular}

Table 6

The impact of features descriptors on the performance of AOA against other recent optimizers over Selection ratio measures

\begin{tabular}{ccccccccc}
\hline Selection ratio & \multicolumn{3}{c}{ GT dataset } & & \multicolumn{3}{c}{ FEI dataset } \\
\cline { 2 - 4 } \cline { 7 - 9 } Algorithms & HOG & LBP & GLCM & & HOG & LBP & GLCM \\
\hline HHO & 0.5306 & 0.5714 & 0.5714 & & 0.5714 & 0.6122 & 0.3878 \\
SCA & 0.3673 & $\mathbf{0 . 2 0 4 1}$ & $\mathbf{0 . 2 4 4 9}$ & & $\mathbf{0 . 2 2 4 5}$ & $\mathbf{0 . 2 0 4 1}$ & $\mathbf{0 . 2 4 4 9}$ \\
EO & 0.3878 & 0.5306 & 0.3673 & & 0.3673 & 0.4898 & 0.3673 \\
EPO & 0.3673 & 0.2449 & 0.4082 & & 0.3673 & 0.2449 & 0.3673 \\
MRFO & 0.8776 & 0.5714 & 0.5306 & & 0.5714 & 0.4898 & 0.3673 \\
HGSO & 0.5306 & 0.4898 & 0.5102 & & 0.4490 & 0.5714 & 0.5306 \\
MVO & 0.4898 & 0.4898 & 0.5306 & & 0.6122 & 0.6327 & 0.5102 \\
AOA & $\mathbf{0 . 2 8 5 7}$ & 0.2449 & 0.5510 & & 0.3469 & 0.6327 & 0.4694 \\
\hline
\end{tabular}




\section{Table 7}

The impact of features descriptors on the performance of AOA against other recent optimizers over Recall measures

\begin{tabular}{cccccccc}
\hline Recall & \multicolumn{3}{c}{ GT dataset } & & \multicolumn{3}{c}{ FEI dataset } \\
\cline { 2 - 4 } Algorithms & HOG & LBP & GLCM & & HOG & LBP & GLCM \\
\hline HHO & 0.9040 & 0.8747 & 0.9000 & & 0.9875 & 0.9425 & 0.9900 \\
SCA & 0.8960 & 0.8693 & 0.8907 & & 0.9850 & $\mathbf{0 . 9 5 5 0}$ & 0.9900 \\
EO & 0.9067 & 0.8693 & 0.8987 & & 0.9925 & 0.9475 & 0.9900 \\
EPO & 0.9040 & $\mathbf{0 . 8 8 1 3}$ & 0.8960 & & 0.9875 & 0.9400 & 0.9825 \\
MRFO & $\mathbf{0 . 9 1 0 7}$ & 0.8707 & 0.8973 & & $\mathbf{0 . 9 9 5 0}$ & 0.9500 & 0.9925 \\
HGSO & 0.9000 & 0.8613 & 0.8880 & & 0.9925 & 0.9400 & $\mathbf{0 . 9 9 5 0}$ \\
MVO & 0.9080 & 0.8693 & 0.9040 & & 0.9925 & 0.9450 & 0.9925 \\
AOA & 0.9040 & 0.8733 & $\mathbf{0 . 9 0 8 0}$ & & $\mathbf{0 . 9 9 5 0}$ & 0.9475 & $\mathbf{0 . 9 9 5 0}$ \\
\hline
\end{tabular}

Table 8

The impact of features descriptors on the performance of AOA against other recent optimizers over Precision measures

\begin{tabular}{ccccccccc}
\hline Precision & \multicolumn{3}{c}{ GT dataset } & & \multicolumn{3}{c}{ FEI dataset } \\
\cline { 2 - 4 } \cline { 7 - 9 } Algorithms & HOG & LBP & GLCM & & HOG & LBP & GLCM \\
\hline HHO & 0.9014 & $\mathbf{0 . 8 6 7 7}$ & 0.8949 & & 0.9903 & 0.9347 & 0.9951 \\
SCA & 0.8891 & 0.8400 & 0.8870 & & 0.9880 & 0.9560 & 0.9877 \\
EO & 0.9018 & 0.8414 & 0.8927 & & 0.9928 & $\mathbf{0 . 9 6 4 2}$ & 0.9904 \\
EPO & 0.8960 & 0.8475 & 0.8883 & & 0.9882 & 0.9383 & 0.9881 \\
MRFO & 0.9052 & 0.8352 & 0.8913 & & $\mathbf{0 . 9 9 5 1}$ & 0.9510 & 0.9929 \\
HGSO & 0.8908 & 0.8361 & 0.8874 & & 0.9928 & 0.9417 & 0.9927 \\
MVO & 0.8974 & 0.8399 & 0.8977 & & 0.9927 & 0.9478 & 0.9927 \\
AOA & $\mathbf{0 . 9 0 9 9}$ & 0.8529 & $\mathbf{0 . 8 9 8 0}$ & & $\mathbf{0 . 9 9 5 1}$ & 0.9457 & $\mathbf{0 . 9 9 5 1}$ \\
\hline
\end{tabular}

77 and 8. By inspecting the results of precision measure, we can see that AOA and MRFO based HOG for Fei dataset provide the same performance with $99.50 \%$ as precision. Also, HGSO and

From Table 8, AOA-based HOG and GLCM provide better performance compared to other optimizers for both datasets. Moroever, MRFO based HOG provides the same performance for FEI as AOA-based HOG, whereas it takes the second rank for the GT dataset.

In terms of F-score : Table 9 indicates the values of F-score obtained by AOA and other optimizers by employing three descriptors features like HOG, LBP and GLCM for both datasets. By inspecting the obtained results, we can see that F-score values over FEI are significantly higher than than GTdataset due to the balanced samples of gender categories. For the GT dataset, a great competition between MRFO and AOA-based HOG is highlighted with a slight advantage for MRFO because the margin is significantly lower with a value of 0.0008. In addition, AOAbased GLCM still better for GT datasets than other algorithms including, HHO, SCA, EO, EPO, MRFO, HGSO and MVO. Also, wrapper FS techniques based on LBP show lower values of F-score compared to others descriptors for both datasets (GT \& FEI).

For FEI, AOA and MRFO based HOG demonstrate a strong efficiency of Fscore with 99.50\% as performance compared to other algorithms as HHO, SCA, EO, EPO, HGSO and MVO.Also, 


\section{Table 9}

The impact of features descriptors on the performance of AOA against other recent optimizers over F-score measures

\begin{tabular}{|c|c|c|c|c|c|c|}
\hline \multirow{2}{*}{$\begin{array}{c}\text { F-score } \\
\text { Algorithms }\end{array}$} & \multicolumn{3}{|c|}{ GT dataset } & \multicolumn{3}{|c|}{ FEI dataset } \\
\hline & $\mathrm{HOG}$ & LBP & GLCM & $\mathrm{HOG}$ & LBP & GLCM \\
\hline $\mathrm{HHO}$ & 0.8977 & 0.8427 & 0.8953 & 0.9887 & 0.9379 & 0.9924 \\
\hline SCA & 0.8892 & 0.8428 & 0.8866 & 0.9862 & 0.9549 & 0.9888 \\
\hline $\mathrm{EO}$ & 0.9026 & 0.8400 & 0.8922 & 0.9925 & 0.9547 & 0.9900 \\
\hline $\mathrm{EPO}$ & 0.8981 & 0.8496 & 0.8898 & 0.9875 & 0.9388 & 0.9848 \\
\hline MRFO & 0.9058 & 0.8328 & 0.8896 & 0.9950 & 0.9499 & 0.9925 \\
\hline HGSO & 0.8918 & 0.8359 & 0.8854 & 0.9925 & 0.9397 & 0.9938 \\
\hline MVO & 0.9004 & 0.8351 & 0.8967 & 0.9925 & 0.9449 & 0.9925 \\
\hline $\mathrm{AOA}$ & 0.9050 & 0.8422 & 0.9009 & 0.9950 & 0.9453 & 0.9950 \\
\hline
\end{tabular}

\subsubsection{Statistical analysis}

To validate the efficiency of AOA to other competitive algorithms, a statistical study is required. Thus, this study is validated by Wilcoxon ranksum test between the fitness values obtained by AOA and other algorithms including HHO, SCA, EO, EPO, MRFO, HGSO, and MVO. From Table. 10, we can observe for both datasets that AOA is statistically significant to all competitors in the case of the GLCM descriptor. Also, the same behavior is highlighted for the FEI dataset when the descriptor is HOG. Additionally, AOA is not significant to MVO based HOG and EPObased LBP in the case of the GT datasets. Also, EO-based LBP in the case of the FEI dataset dominates AOA. In general, AOA shows a good performance in terms of Wilcoxon's test.

Table 10

Statistical study using Wilcoxon's test

\begin{tabular}{ccccccccc}
\hline AOA & \multicolumn{3}{c}{ GT dataset } & & \multicolumn{3}{c}{ FEI dataset } \\
\cline { 2 - 4 } \cline { 6 - 8 } versus & HOG & LBP & GLCM & & HOG & LBP & GLCM \\
\hline HHO & $4.40 \mathrm{E}-21$ & $2.06 \mathrm{E}-14$ & $5.00 \mathrm{E}-02$ & & $1.26 \mathrm{E}-32$ & $3.59 \mathrm{E}-30$ & $1.23 \mathrm{E}-39$ \\
SCA & $2.20 \mathrm{E}-02$ & $1.71 \mathrm{E}-02$ & $6.86 \mathrm{E}-31$ & & $9.74 \mathrm{E}-27$ & $1.40 \mathrm{E}-04$ & $2.62 \mathrm{E}-38$ \\
EO & $7.20 \mathrm{E}-03$ & $3.37 \mathrm{E}-02$ & $1.50 \mathrm{E}-05$ & & $2.70 \mathrm{E}-13$ & $\mathbf{2 . 6 4 E}-01$ & $1.33 \mathrm{E}-38$ \\
EPO & $1.04 \mathrm{E}-02$ & $\mathbf{8 . 0 9 E}-01$ & $6.97 \mathrm{E}-21$ & & $8.87 \mathrm{E}-23$ & $2.86 \mathrm{E}-17$ & $7.37 \mathrm{E}-42$ \\
MRFO & $5.11 \mathrm{E}-07$ & $2.38 \mathrm{E}-10$ & $1.13 \mathrm{E}-06$ & & $7.25 \mathrm{E}-19$ & $1.91 \mathrm{E}-05$ & $5.49 \mathrm{E}-39$ \\
HGSO & $3.58 \mathrm{E}-25$ & $5.65 \mathrm{E}-26$ & $1.11 \mathrm{E}-36$ & & $1.15 \mathrm{E}-19$ & $4.36 \mathrm{E}-30$ & $1.08 \mathrm{E}-39$ \\
MVO & $\mathbf{9 . 9 1 E - 0 2}$ & $2.20 \mathrm{E}-04$ & $3.16 \mathrm{E}-17$ & & $1.11 \mathrm{E}-19$ & $1.30 \mathrm{E}-17$ & $1.31 \mathrm{E}-40$ \\
\hline
\end{tabular}

\subsubsection{Graphical analysis}

The fitness curves obtained by the different optimizers are shown in the Figure. 7. By analyzing the behavior of the convergence of the AOA algorithm for the two databases based on the different descriptors, a clear growth is illustrated by increasing the number of iterations compared to other algorithms, including EO, MVO, EPO, MRFO, HHO, SCA, and HGSO. This phenomenon is justified by a better balance between exploitation and exploration, making it possible to avoid convergence towards local minima. For both datasets, we can conclude that AOA based on the 


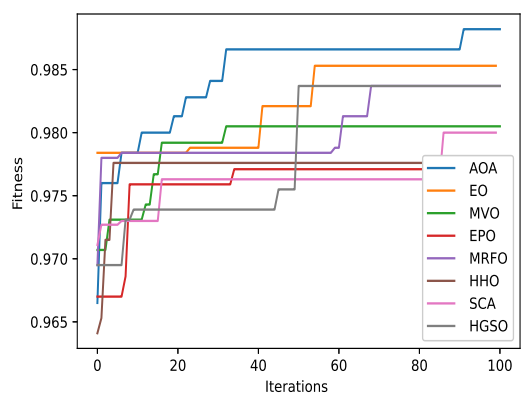

(a) HOG-FEI

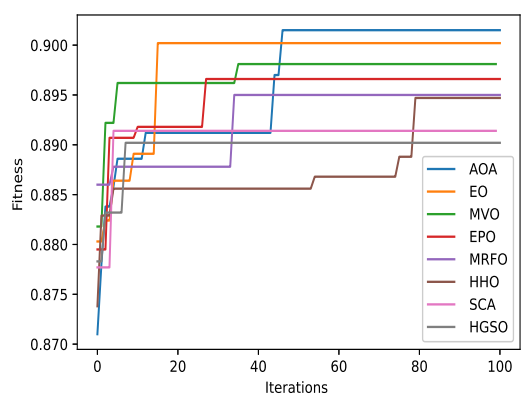

(d) HOG-GT

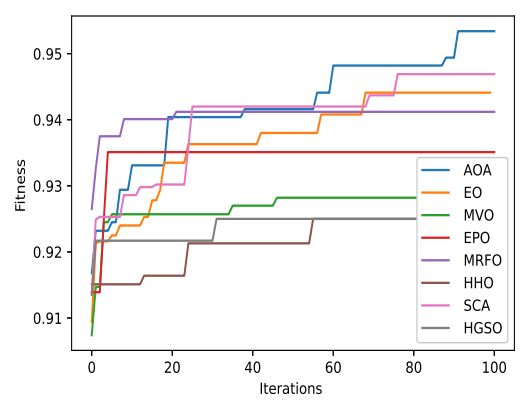

(b) LBP-FEI

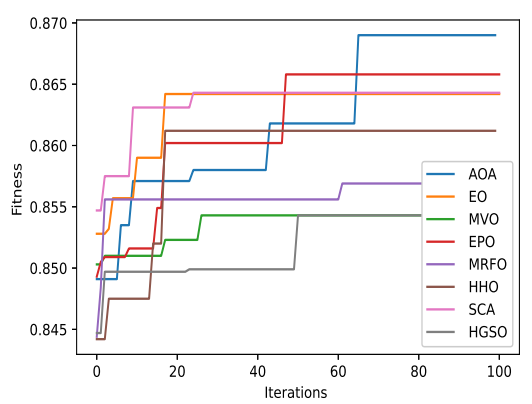

(e) LBP-GT

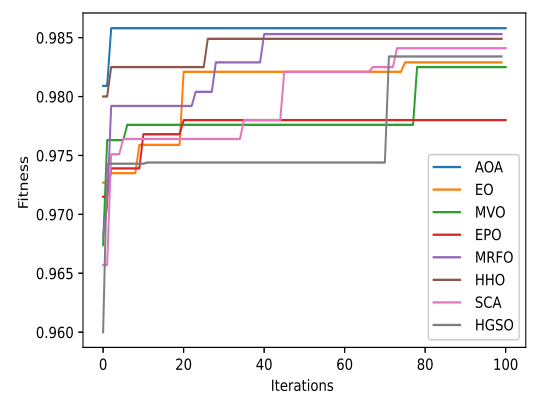

(c) GLCM-FEI

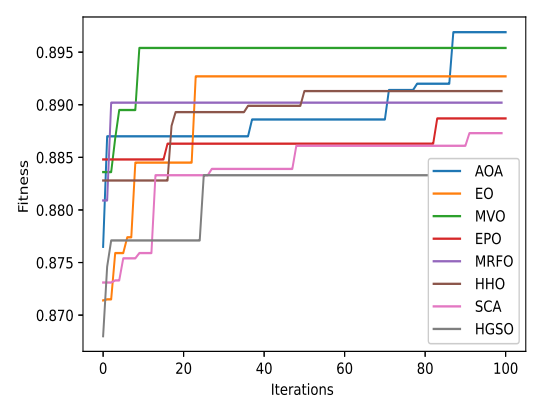

(f) GLCM-GT

Figure 7: Convergence curve of AOA versus other swarm intelligence algorithms over all datasets

HOG descriptor provides a higher value of fitness compared to other descriptors as GLCM and LBP. Also, AOA highlights a superiority to other competitors, including EO, MVO, EPO, MRFO, HHO, SCA, and HGSO.

In addition, we have graphically represented the ROC curve, as shown in Figure. 8, which relates True Positive Rate (TPR) as a function of False Positive Rate (FPR) by using the different algorithms based on the three descriptors (HOG, LBP, and GLCM) for all the images of the two corpora (FEI and GT). We notice that AOA shows a clear performance in terms of AUC compared to other optimizers. For the FEI dataset, the values of AUC obtained by AOA-based on HOG, LBPn and GLCM are 0.9951, 0.9606, and 0.9951, respectively. However for the GT dataset obtained $0.9099,0.8529$, and 0.9027 , respectively.

From the obtained results, we notice that the gender performance of GT dataset causes less quality compared to FET and this is due to the complexity of the datasets which depends on several challenging factors such as highlighting variation, facial expressions, different pose and occluded eye area.

\subsection{Comparative study with the existing works}

A novel comparative study with the literature works has been realized further to explore the power of the proposed system AOA-based HOG. Mainly, we have selected some works from literature based on machine learning, deep learning, and genetic-based FS. It is essential to show that the comparison is tough because the most authors used different conditions

- FEI dataset: A deep analysis of the Table. 11, indicates that the proposed method AOAbased on Multi blocks HOG with BPNN as classifier achieves a higher performance of accuracy i.e., $99.16 \%$ as correct prediction rate of gender from faces. The majority of machine learning methods used mainly SVM classifier with multi-features based on handcrafted techniques inspired 


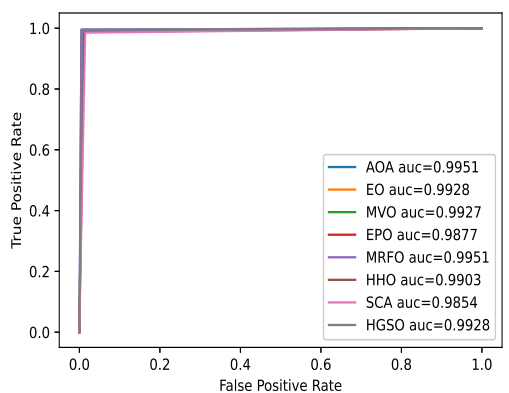

(a) HOG-FEI

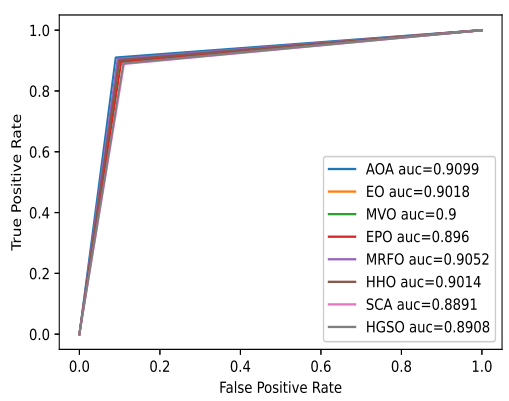

(d) HOG-GT

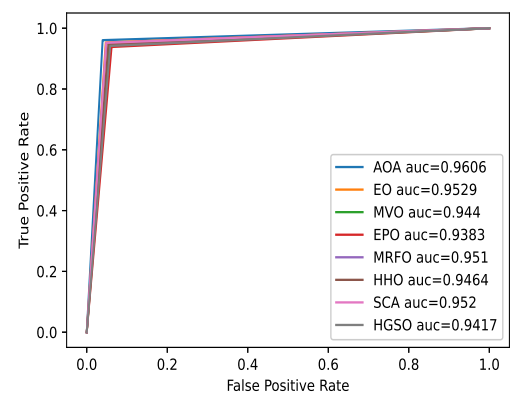

(b) LBP-FEI

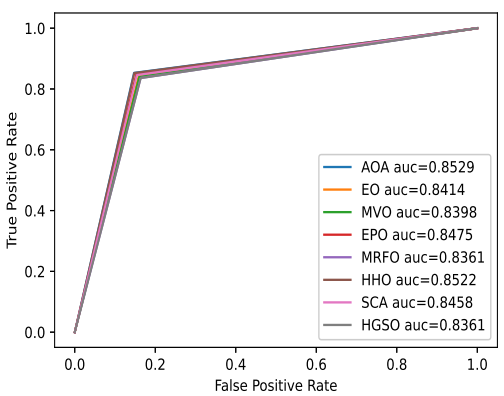

(e) LBP-GT

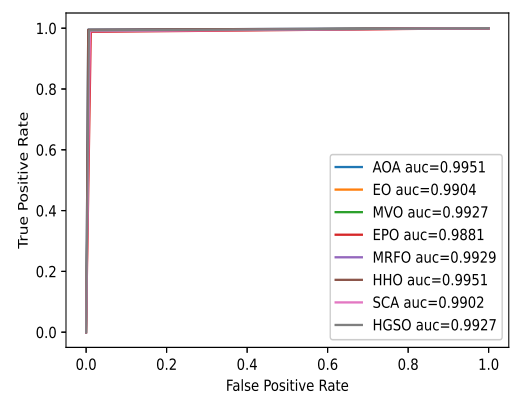

(c) GLCM-FEI

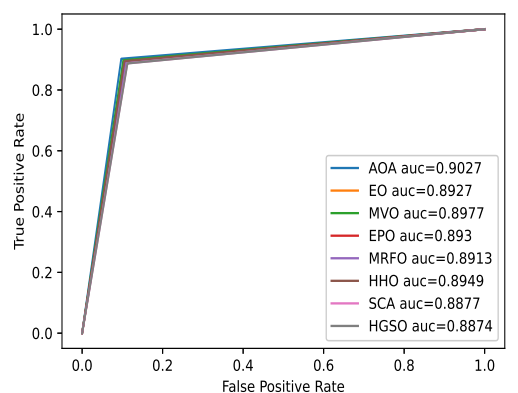

(f) GLCM-GT

Figure 8: ROC of AOA versus other swarm intelligence algorithms over all datasets

by LBP, LDP, and HOG. In this category, the SVM proposed by 8 represents the best classifier that can predict the gender correctly with high accuracy of $99 \%$ compared to other ML methods. The Deep-gender provided by [67] shows a good accuracy with $98.75 \%$. Furthermore, a wrapper FS based on a genetic algorithm is employed for predicting gender from the face, which used Eigen-space of features. The authors utilized BPNN as a classifier, where GA is used for selecting the significant Eigen vectors, which obtained $96 \%$ as accuracy. In conclusion, AOA-based HOG and AOA-based GLCM are the best classifier for predicting gender from face compared to other approaches inspired by ML and deep CNN.

- GT dataset: For this dataset, a novel execution is realized due to the value of $k$-folds. We note that some works of literature used 2-folds. So, Table. 12 highlights the performance results of AOA with some methods of machine learning (ML) methods using 2-folds across the correct rate of gender identification.

The obtained results in terms of accuracy indicate that the AOA-based multi-blocks HOG provides a significant superiority compared to other ML methods such as SVM based on combined DWT with DCT. Also, it is essential to show that the Multi blocks LBP based-AOA reached a lower performance with $97.25 \%$ in terms of the correct rate of gender identification. The AOAbased Multi blocks GLCM is ranked in the second position with $99.15 \%$ of accuracy, followed by the work of [81], which applied SVM based on DCT and reached $98.96 \%$ in accuracy.

\section{Conclusion}

This paper presents a novel wrapper FS-based AOA for identifying the facial gender classes. For this, we have integrated three textural descriptors, including HOG, LBP, and GLCM tested over two datasets (GT \& FEI). 


\section{Table 11}

Comparative performance in terms of accuracy with the existing approaches-FEI dataset.

\begin{tabular}{|c|c|c|c|}
\hline References & classifier & extracted features & Accuracy \\
\hline [7] & SVM & $\mathrm{DRLBP}++\mathrm{RILPQ}+\mathrm{PHOG}$ & $95.30 \%$ \\
\hline 8 & SVM & 8-LDP $+\mathrm{LBP}$ & $99 \%$ \\
\hline 58 & LDA + weighting vote & Intensity of lower part of face & $94 \%$ \\
\hline 67 & Deepgender & $*$ & $98.75 \%$ \\
\hline 55 & GA-BPNN & Eigen-features based on PCA & $96 \%$ \\
\hline 79 & MSFS-CRFs & Segmentation based on Super-Pixels & $93.70 \%$ \\
\hline 80 & SVM & Multi-features (BoW+SIFT) & $98 \%$ \\
\hline \multirow{3}{*}{ Proposed method } & \multirow{3}{*}{ AOA-BPNN } & Multi blocks HOG & $99.16 \%$ \\
\hline & & Multi blocks LBP & $95.61 \%$ \\
\hline & & Multi blocks GLCM & $99.04 \%$ \\
\hline
\end{tabular}

Table 12

Comparative performance in terms of accuracy with the existing methods- GT dataset

\begin{tabular}{|c|c|c|c|}
\hline References & classifier & extracted features & Accuracy \\
\hline$[81$ & SVM (2-folds) & DCT & $98.96 \%$ \\
\hline$[1]$ & SVM (2-folds) & KPCA & $97.38 \%$ \\
\hline$[82$ & SVM (2-folds) & DWT+DCT & $99 \%$ \\
\hline \multirow{3}{*}{ Proposed method } & \multirow{2}{*}{ AOA-BPNN (2-folds) } & Multi blocks HOG & $\mathbf{9 9 . 5 0 \%}$ \\
\cline { 3 - 4 } & & Multi blocks LBP & $97.25 \%$ \\
\cline { 3 - 4 } & & Multi blokcs GLCM & $99.15 \%$ \\
\hline
\end{tabular}


The obtained results showed the high performance of AOA-based HOG for both datasets in terms of accuracy and F-score for both datasets. Also, SCA allows keeping the smallest number of relevant blocks with a speed time. As the advantage of the proposed method, the AOA ensures a good balance between the most relevant gender features from faces and the correct rate of gender classification. However, some drawbacks can be highlighted of AOA and the handcrafted methods, and mainly they are several parameters that are defined randomly in the initialization steps of AOA. Also, the number of blocks for each handcrafted descriptor is fixed to $7 \times 7$ which has a higher impact on the performance. Furthermore GLCM, required to define two parameters: the displacement $(d)$ and orientation $(\theta)$. All parameters can be tuned automatically as hyper-heuristic AOA in the future.

The new horizon can be explored, like the automatic fusion between textural descriptors assisted by FS wrapper using an improved version of AOA or another recent swarm algorithm which required fewer parameters with fast convergence. Also, the combination of handcrafted methods with deep features is conceivable and promising in automatic facial analysis, particularly gender recognition and age prediction or recognition of emotions.

\section{Compliance with Ethical Standards}

- Conflict of interest: The authors have declared that there is no conflict of interest.

- Funding: The authors declare that they are not involved in any financial or other partnerships.

- Ethical approval: This article does not contain any studies with human participants or animals performed by any of the authors.

- Informed consent was obtained from all individual participants included in the study

\section{Author contributions}

I. Neggaz realized the idea of facial analysis using AOA. Also, she wrote the different parts of the paper. Pr H. FIZAZI checked the methodology and the quality of the current work. The authors declare that numerous operations have been carried out, beginning with the creation of language editing and plagiarism detection software.

\section{References}

[1] A. Goel, V. P. Vishwakarma, Gender classification using kpca and svm, in: 2016 IEEE International Conference on Recent Trends in Electronics, Information Communication Technology (RTEICT), 2016, pp. 291-295. doi:doi:10.1109/RTEICT.2016.7807829.

[2] T. K. Sajja, H. K. Kalluri, Gender classification based on face images of local binary pattern using support vector machine and back propagation neural networks, Advances in Modelling and Analysis B 62 (2019) 31-35.

[3] H.-T. Nguyen, T. T. N. Huong, Gender classification by lpq features from intensity and monogenic images, in: 2017 4th NAFOSTED Conference on Information and Computer Science, IEEE, 2017, pp. 96-100.

[4] S. M. Osman, S. Viriri, Dynamic local ternary patterns for gender identification using facial components, in: International Conference on Computer Vision and Graphics, Springer, 2020, pp. 133-141. 
[5] T. KHALIFA, G. ŞENGÜL, Gender prediction from facial images using local binary patterns and histograms of oriented gradients transformations, Niğde Ömer Halisdemir Üniversitesi Mühendislik Bilimleri Dergisi 7 (2018) 14-22.

[6] S. E. Bekhouche, A. Ouafi, F. Dornaika, A. Taleb-Ahmed, A. Hadid, Pyramid multi-level features for facial demographic estimation, Expert Systems with Applications 80 (2017) 297310 .

[7] A. A. Micheal, P. Geetha, Combined feature extraction for multi-view gender recognition, in: Smart Intelligent Computing and Applications, Springer, 2019, pp. 219-228.

[8] A. Geetha, M. Sundaram, B. Vijayakumari, Gender classification from face images by mixing the classifier outcome of prime, distinct descriptors, soft computing 23 (2019) 2525-2535.

[9] A. Singh, N. Rai, P. Sharma, P. Nagrath, R. Jain, Age, gender prediction and emotion recognition using convolutional neural network, Available at SSRN 3833759 (2021).

[10] A. Peimankar, S. Puthusserypady, Dens-ecg: A deep learning approach for ecg signal delineation, Expert Systems with Applications 165 (2021) 113911.

[11] H. Yu, L. T. Yang, Q. Zhang, D. Armstrong, M. J. Deen, Convolutional neural networks for medical image analysis: state-of-the-art, comparisons, improvement and perspectives, Neurocomputing (2021).

[12] M. Peker, Classification of hyperspectral imagery using a fully complex-valued wavelet neural network with deep convolutional features, Expert Systems with Applications 173 (2021) 114708 .

[13] H. Alhichri, A. S. Alswayed, Y. Bazi, N. Ammour, N. A. Alajlan, Classification of remote sensing images using efficientnet-b3 cnn model with attention, IEEE Access 9 (2021) 1407814094 .

[14] H. T. Huynh, H. Nguyen, Joint age estimation and gender classification of asian faces using wide resnet, SN Computer Science 1 (2020) 1-9.

[15] A. V. Savchenko, Efficient facial representations for age, gender and identity recognition in organizing photo albums using multi-output convnet, PeerJ Computer Science 5 (2019) e197.

[16] S. Lapuschkin, A. Binder, K.-R. Muller, W. Samek, Understanding and comparing deep neural networks for age and gender classification, in: Proceedings of the IEEE International Conference on Computer Vision Workshops, 2017, pp. 1629-1638.

[17] D. P. d. Silva, Age and gender classification: a proposed system, Ph.D. thesis, 2019.

[18] B. Abirami, T. Subashini, V. Mahavaishnavi, Gender and age prediction from real time facial images using cnn, Materials Today: Proceedings 33 (2020) 4708-4712.

[19] C.-J. Lin, Y.-C. Li, H.-Y. Lin, Using convolutional neural networks based on a taguchi method for face gender recognition, Electronics 9 (2020) 1227.

[20] A. Swaminathan, M. Chaba, D. K. Sharma, Y. Chaba, Gender classification using facial embeddings: A novel approach, Procedia Computer Science 167 (2020) 2634-2642. 
[21] A. Greco, A. Saggese, M. Vento, V. Vigilante, Gender recognition in the wild: a robustness evaluation over corrupted images, Journal of Ambient Intelligence and Humanized Computing (2020) $1-12$.

[22] J. H. Holland, Genetic algorithms, Scientific american 267 (1992) 66-73.

[23] R. Storn, K. Price, Differential evolution-a simple and efficient heuristic for global optimization over continuous spaces, Journal of global optimization 11 (1997) 341-359.

[24] G. Rudolph, Evolution strategies, Evolutionary computation 1 (2000) 81-88.

[25] T. Bäck, F. Hoffmeister, H.-P. Schwefel, A survey of evolution strategies, in: Proceedings of the fourth international conference on genetic algorithms, Citeseer, 1991.

[26] J. R. Koza, J. R. Koza, Genetic programming: on the programming of computers by means of natural selection, volume 1, MIT press, 1992.

[27] R. Eberhart, J. Kennedy, A new optimizer using particle swarm theory, in: MHS'95. Proceedings of the Sixth International Symposium on Micro Machine and Human Science, Ieee, 1995, pp. 39-43.

[28] D. Karaboga, B. Akay, A comparative study of artificial bee colony algorithm, Applied mathematics and computation 214 (2009) 108-132.

[29] S. Mirjalili, S. M. Mirjalili, A. Lewis, Grey wolf optimizer, Advances in engineering software 69 (2014) 46-61.

[30] A. A. Heidari, S. Mirjalili, H. Faris, I. Aljarah, M. Mafarja, H. Chen, Harris hawks optimization: Algorithm and applications, Future generation computer systems 97 (2019) 849-872.

[31] S. Mirjalili, A. Lewis, The whale optimization algorithm, Advances in engineering software 95 (2016) 51-67.

[32] S. Mirjalili, A. H. Gandomi, S. Z. Mirjalili, S. Saremi, H. Faris, S. M. Mirjalili, Salp swarm algorithm: A bio-inspired optimizer for engineering design problems, Advances in Engineering Software 114 (2017) 163-191.

[33] S. Saremi, S. Mirjalili, A. Lewis, Grasshopper optimisation algorithm: theory and application, Advances in Engineering Software 105 (2017) 30-47.

[34] S. Mirjalili, The ant lion optimizer, Advances in engineering software 83 (2015) 80-98.

[35] G. Dhiman, V. Kumar, Emperor penguin optimizer: a bio-inspired algorithm for engineering problems, Knowledge-Based Systems 159 (2018) 20-50.

[36] W. Zhao, Z. Zhang, L. Wang, Manta ray foraging optimization: An effective bio-inspired optimizer for engineering applications, Engineering Applications of Artificial Intelligence 87 (2020) 103300.

[37] F. A. Hashim, K. Hussain, E. H. Houssein, M. S. Mabrouk, W. Al-Atabany, Archimedes optimization algorithm: a new metaheuristic algorithm for solving optimization problems, Applied Intelligence (2020) 1-21. 
[38] A. Faramarzi, M. Heidarinejad, B. Stephens, S. Mirjalili, Equilibrium optimizer: A novel optimization algorithm, Knowledge-Based Systems 191 (2020) 105190.

[39] S. Mirjalili, S. M. Mirjalili, A. Hatamlou, Multi-verse optimizer: a nature-inspired algorithm for global optimization, Neural Computing and Applications 27 (2016) 495-513.

[40] F. A. Hashim, E. H. Houssein, M. S. Mabrouk, W. Al-Atabany, S. Mirjalili, Henry gas solubility optimization: A novel physics-based algorithm, Future Generation Computer Systems 101 (2019) 646-667.

[41] C. Zhang, X. Li, L. Gao, Q. Wu, An improved electromagnetism-like mechanism algorithm for constrained optimization, Expert Systems with Applications 40 (2013) 5621-5634.

[42] A. Kaveh, A. Dadras, A novel meta-heuristic optimization algorithm: thermal exchange optimization, Advances in Engineering Software 110 (2017) 69-84.

[43] I. Ahmadianfar, A. A. Heidari, A. H. Gandomi, X. Chu, H. Chen, Run beyond the metaphor: An efficient optimization algorithm based on runge kutta method, Expert Systems with Applications (2021) 115079.

[44] S. Mirjalili, Sca: a sine cosine algorithm for solving optimization problems, Knowledge-based systems 96 (2016) 120-133.

[45] Y. Yang, H. Chen, A. A. Heidari, A. H. Gandomi, Hunger games search: Visions, conception, implementation, deep analysis, perspectives, and towards performance shifts, Expert Systems with Applications (2021) 114864.

[46] R. Moghdani, K. Salimifard, Volleyball premier league algorithm, Applied Soft Computing 64 (2018) 161-185.

[47] K. K. Ghosh, R. Guha, S. K. Bera, N. Kumar, R. Sarkar, S-shaped versus v-shaped transfer functions for binary manta ray foraging optimization in feature selection problem, Neural Computing and Applications (2021) 1-15.

[48] G. Dhiman, D. Oliva, A. Kaur, K. K. Singh, S. Vimal, A. Sharma, K. Cengiz, Bepo: a novel binary emperor penguin optimizer for automatic feature selection, Knowledge-Based Systems 211 (2021) 106560.

[49] T. Thaher, A. A. Heidari, M. Mafarja, J. S. Dong, S. Mirjalili, Binary harris hawks optimizer for high-dimensional, low sample size feature selection, in: Evolutionary machine learning techniques, Springer, 2020, pp. 251-272.

[50] Q. Al-Tashi, H. M. Rais, S. J. Abdulkadir, S. Mirjalili, H. Alhussian, A review of grey wolf optimizer-based feature selection methods for classification, Evolutionary Machine Learning Techniques (2020) 273-286.

[51] M. Mafarja, S. Mirjalili, Whale optimization approaches for wrapper feature selection, Applied Soft Computing 62 (2018) 441-453.

[52] N. Neggaz, E. H. Houssein, K. Hussain, An efficient henry gas solubility optimization for feature selection, Expert Systems with Applications 152 (2020) 113364. 
[53] Y. Gao, Y. Zhou, Q. Luo, An efficient binary equilibrium optimizer algorithm for feature selection, IEEE Access 8 (2020) 140936-140963.

[54] S. Taghian, M. H. Nadimi-Shahraki, Binary sine cosine algorithms for feature selection from medical data, arXiv preprint arXiv:1911.07805 (2019).

[55] Y. Zhou, Z. Li, Facial eigen-feature based gender recognition with an improved genetic algorithm, Journal of Intelligent \& Fuzzy Systems 37 (2019) 4891-4902.

[56] O. Surinta, T. Khamket, Gender recognition from facial images using local gradient feature descriptors, in: 2019 14th International Joint Symposium on Artificial Intelligence and Natural Language Processing (iSAI-NLP), IEEE, 2019, pp. 1-6.

[57] C. Zhang, H. Ding, Y. Shang, Z. Shao, X. Fu, Gender classification based on multiscale facial fusion feature, Mathematical Problems in Engineering 2018 (2018).

[58] B. Ghojogh, S. B. Shouraki, H. Mohammadzade, E. Iranmehr, A fusion-based gender recognition method using facial images, in: Electrical Engineering (ICEE), Iranian Conference on, IEEE, 2018, pp. 1493-1498.

[59] H. K. Omer, H. A. Jalab, A. M. Hasan, N. E. Tawfiq, Combination of local binary pattern and face geometric features for gender classification from face images, in: 2019 9th IEEE International Conference on Control System, Computing and Engineering (ICCSCE), IEEE, 2019, pp. 158-161.

[60] W.-S. Chen, R.-H. Jeng, A new patch-based lbp with adaptive weights for gender classification of human face, Journal of the Chinese Institute of Engineers 43 (2020) 451-457.

[61] S. Pai, R. Shettigar, Gender recognition from face images using sift descriptors and trainable features, in: Advances in Artificial Intelligence and Data Engineering, Springer, 2021, pp. $1173-1186$.

[62] M. Duan, K. Li, C. Yang, K. Li, A hybrid deep learning cnn-elm for age and gender classification, Neurocomputing 275 (2018) 448-461.

[63] A. Acien, A. Morales, R. Vera-Rodriguez, I. Bartolome, J. Fierrez, Measuring the gender and ethnicity bias in deep models for face recognition, in: Iberoamerican Congress on Pattern Recognition, Springer, 2018, pp. 584-593.

[64] K. Ito, H. Kawai, T. Okano, T. Aoki, Age and gender prediction from face images using convolutional neural network, in: 2018 Asia-Pacific Signal and Information Processing Association Annual Summit and Conference (APSIPA ASC), IEEE, 2018, pp. 7-11.

[65] S. Mane, G. Shah, Facial recognition, expression recognition, and gender identification, in: Data Management, Analytics and Innovation, Springer, 2019, pp. 275-290.

[66] B. Agrawal, M. Dixit, Age estimation and gender prediction using convolutional neural network, in: International Conference on Sustainable and Innovative Solutions for Current Challenges in Engineering \& Technology, Springer, 2019, pp. 163-175. 
[67] K. Z. Haider, K. R. Malik, S. Khalid, T. Nawaz, S. Jabbar, Deepgender: real-time gender classification using deep learning for smartphones, Journal of Real-Time Image Processing 16 (2019) 15-29.

[68] F. Simanjuntak, G. Azzopardi, Fusion of cnn- and cosfire-based features with application to gender recognition from face images, in: K. Arai, S. Kapoor (Eds.), Advances in Computer Vision, Springer International Publishing, Cham, 2020, pp. 444-458.

[69] N. Dwivedi, D. K. Singh, Review of deep learning techniques for gender classification in images, in: Harmony Search and Nature Inspired Optimization Algorithms, Springer, 2019, pp. 1089-1099.

[70] A. Althnian, N. Aloboud, N. Alkharashi, F. Alduwaish, M. Alrshoud, H. Kurdi, Face gender recognition in the wild: An extensive performance comparison of deep-learned, hand-crafted, and fused features with deep and traditional models, Applied Sciences 11 (2021) 89.

[71] C.-Y. Hsu, L.-E. Lin, C. H. Lin, Age and gender recognition with random occluded data augmentation on facial images, Multimedia Tools and Applications (2021) 1-23.

[72] T. Ojala, M. Pietikainen, T. Maenpaa, Multiresolution gray-scale and rotation invariant texture classification with local binary patterns, IEEE Transactions on pattern analysis and machine intelligence 24 (2002) 971-987.

[73] N. Dalal, B. Triggs, Histograms of oriented gradients for human detection, in: 2005 IEEE computer society conference on computer vision and pattern recognition (CVPR'05), volume 1, Ieee, 2005, pp. 886-893.

[74] B. T. Hung, Face recognition using hybrid hog-cnn approach, in: Research in Intelligent and Computing in Engineering, Springer, 2021, pp. 715-723.

[75] K. A. Patil, et al., Features and methods of human age estimation: Opportunities and challenges in medical image processing, Turkish Journal of Computer and Mathematics Education (TURCOMAT) 12 (2021) 294-318.

[76] G. Pattnaik, K. Parvathi, Automatic detection and classification of tomato pests using support vector machine based on hog and lbp feature extraction technique, in: Progress in Advanced Computing and Intelligent Engineering, Springer, 2021, pp. 49-55.

[77] D. Lakshmi, R. Ponnusamy, Facial emotion recognition using modified hog and lbp features with deep stacked autoencoders, Microprocessors and Microsystems 82 (2021) 103834.

[78] S. Vimal, Y. H. Robinson, M. Kaliappan, K. Vijayalakshmi, S. Seo, A method of progression detection for glaucoma using k-means and the glcm algorithm toward smart medical prediction, The Journal of Supercomputing (2021) 1-17.

[79] K. Khan, M. Attique, I. Syed, A. Gul, Automatic gender classification through face segmentation, Symmetry 11 (2019) 770.

[80] S. Kumar, S. Singh, J. Kumar, Gender classification using machine learning with multifeature method, in: 2019 IEEE 9th Annual Computing and Communication Workshop and Conference (CCWC), IEEE, 2019, pp. 0648-0653. 
754

[81] A. Goel, V. P. Vishwakarma, Efficient feature extraction using det for gender classification, in: 2016 IEEE International Conference on Recent Trends in Electronics, Information \& Communication Technology (RTEICT), IEEE, 2016, pp. 1925-1928.

[82] A. Goel, V. P. Vishwakarma, Feature extraction technique using hybridization of dwt and dct for gender classification, in: 2016 Ninth International Conference on Contemporary Computing (IC3), IEEE, 2016, pp. 1-6. 